hep-th/0203173

RUNHETC-2002-09

$\mathrm{IMSc} / 2002 / 03 / 01$

CPHT-RR035.0302

\title{
D-branes on Calabi-Yau Manifolds and Superpotentials
}

\author{
Michael R. Douglas ${ }^{1,2,3}$ \\ Suresh Govindarajan ${ }^{4}$ \\ T. Jayaraman ${ }^{5}$ \\ Alessandro Tomasiello 6
}

${ }^{1}$ Department of Physics and Astronomy, Rutgers University, Piscataway, NJ 08855-0849 USA

${ }^{2}$ Isaac Newton Institute for Mathematical Sciences, Cambridge, CB3 0EH, U.K.

${ }^{3}$ I.H.E.S., Le Bois-Marie, Bures-sur-Yvette, 91440 France

${ }^{4}$ Department of Physics, Indian Institute of Technology, Madras, Chennai 600036 India

${ }^{5}$ The Institute of Mathematical Sciences, C.I.T. Campus, Taramani, Chennai 600113 India

${ }^{6}$ Centre de Physique Théorique, Ecole Polytechnique, 91128 Palaiseau, France

Email: mrd@physics.rutgers.edu; suresh@chaos.iitm.ernet.in;

jayaram@imsc.ernet.in; Alessandro.Tomasiello@cpht.polytechnique.fr

We show how to compute terms in an expansion of the world-volume superpotential for fairly general D-branes on the quintic Calabi-Yau using linear sigma model techniques, and show in examples that this superpotential captures the geometry and obstruction theory of bundles and sheaves on this Calabi-Yau.

March 2002

3 Louis Michel Professor 


\section{Introduction}

D-branes on Calabi-Yau manifolds provide a simple and general way to embed $\mathcal{N}=$ 1 supersymmetric gauge theories in string compactifications, and are the starting point for many applications: the general study of $\mathcal{N}=1$ compactification and duality, model building, geometric engineering of gauge theory, AdS/CFT with $\mathcal{N}=1$ supersymmetry, and the study of BPS states.

Much of this work, starting with [16], has focused on D-branes on noncompact CalabiYaus, and one starts to feel that that this case is reasonably well understood. The same ideas should apply just as well to compact Calabi-Yaus, and this holds out the possibility of a much more complete understanding of $\mathcal{N}=1$ compactification than we have now.

At present three approaches to this problem have shown some success. First, for the special case of elliptically fibered Calabi-Yau, one can do T-duality on the fiber, reducing much (but not all) of the problem to the better understood theory of holomorphic curves and surfaces in a CY [18]. Another approach is to study the theory of special Lagrangian submanifolds on the mirror CY; this is also based on T-duality, as has been explained in various ways 45, 35,28.

The third approach grew out of the study of boundary states in Gepner models [40], which gave an explicit construction of certain D-branes in a highly stringy and a priori nongeometric regime: in linear sigma model terms, the Landau-Ginzburg (LG) phase. Arguments from topological open string theory, formalized in the "decoupling statement" of [6], suggest that each B type brane corresponds to a specific holomorphic bundle on the $\mathrm{CY}$ in the large volume limit, so the problem of identifying which bundle corresponds to which boundary state is well-posed.

In 15, 12 it was found that these boundary states could be largely understood in terms of a standard construction of sheaves on projective space $\mathbb{P}^{n}$ formulated by Beilinson [3]. Beilinson's construction links algebraic and geometric data very tightly, and seems to be the best general construction which emerged from much mathematical work on the subject. Following relevant mathematical work on the generalized McKay correspondence [41], this has been generalized to subvarieties of more general toric varieties, in [12, 21, 33, 47 .

Somewhat surprisingly, these constructions lead not just to sheaves but to arbitrary objects in the derived category of coherent sheaves. If one's goal is to study bundles, this is a disadvantage, as one must then work to prove that the object of interest is a bundle. However, there is by now a lot of evidence that physical BPS branes are not just bundles 
or even sheaves, but actually do correspond to objects in the derived category [14. The usual association with bundles and sheaves is only appropriate in the large volume limit, while the formalism of the derived category applies in the stringy regime as well. The physical question is not whether the objects of interest are bundles, but whether they are stable.

To summarize what has been accomplished in this approach so far, we can derive explicit $\mathcal{N}=1$ supersymmetric field theories whose moduli spaces are the classical (open string) moduli spaces of superstring compactification on Calabi-Yau, given an important proviso: it must turn out that sheaves on the ambient space, say $\mathbb{P}^{n}$, in which the CalabiYau of interest is embedded, are the same or at least very similar to sheaves on the embedded Calabi-Yau. The usefulness of this construction depends very much on this point.

At first sight one might be discouraged by examples in low dimension. For example, one might try to study bundles on the torus $T^{2}$ by realizing it as a curve embedded in $\mathbb{P}^{2}$. In fact this would be a very bad idea: the classification of bundles on $\mathbb{P}^{2}$ is very much more complicated than that on $T^{2}$, and the moduli of bundles on $T^{2}$ (for example of a flat connection on $T^{2}$ ) are typically not even present before the restriction.

However, some more thinking about this point shows that this type of construction gets better in higher dimensions, and becomes very useful precisely when one reaches dimension three, i.e. the case of interest. We will tackle the problem from two different but complementary approaches, with roots in mathematics and physics.

Our first approach will use the mathematics of restriction of sheaves. Thus, one first asks: when do two sheaves $E$ and $E^{\prime}$ on the ambient space restrict to the same sheaf on the CY? This can be studied systematically using long exact sequences (or related constructions) as we discuss in section 2 and 3, and one indeed finds that in higher dimension this is generally not a difficult issue. One then asks, how many moduli are present for Dbrane configurations after restriction to the CY? The basic result here is, that on general grounds, linearized moduli correspond to elements of $\operatorname{Ext}^{1}(E, E)$ and will arise as the Serre duals of elements of $\operatorname{Ext}^{d-1}(E, E)$ on the ambient space; again these are under control.

One then needs to know when such linearized moduli correspond to true moduli, i.e. whether they can be given finite vevs. Mathematically this is a complicated problem of obstruction theory, but it is here that physics provides a big advantage: in principle the answer to all such questions is encoded in the superpotential $\mathcal{W}$ of the world-volume 
$\mathcal{N}=1$ theory; allowed finite values of the moduli are the ones which satisfy the F-flatness conditions $\mathcal{W}^{\prime}=0$.

Furthermore, one has techniques for computing the superpotential in interesting models. The leading term has been computed in both the Gepner model [7] and the linear sigma model [12]; it is cubic and agrees with what is needed to reproduce the Beilinson construction. This cubic superpotential can be understood from the geometry of orbifold resolution, in the same way as for the solved case of $\mathbb{C}^{3}$ orbifolds. However, while the superpotential for the noncompact orbifold is exactly cubic, in the LG model the world-sheet superpotential leads to higher order corrections.

In this work, we compute the leading such correction for the quintic, and show how it fits with the geometric interpretation of B branes as bundles on CY (or objects in the derived category).

In section 2, we discuss some examples on the quintic which demonstrate the need for these corrections. One could in principle compute them by perturbation theory in holomorphic Chern-Simons theory; we use this to give an expression for the next to leading order term, at sixth order. This expression is not completely explicit, depending on complicated geometric data such as the Green function for the Laplacian on the CY, but the general form of the expression is clear. We show that, given some plausible assumptions, this turns out to be enough to determine the term. We then show in examples that it plays the anticipated role.

In section 3, we discuss an alternate mathematical approach to getting these corrections, using spectral sequences.

In section 4, we give background on the LG description and use it to compute the sixth order correction explicitly. It takes precisely the form predicted by the geometric analysis.

In sections 5 and 6 , we discuss a number of other examples, further directions and connections with mathematics.

\section{Geometry of sheaves on the quintic}

As discussed in the introduction, the problem of describing the holomorphic structure of $\mathrm{B}$ branes on the quintic can be approached from several directions. In this section we consider them as coherent sheaves, or objects in the derived category of these.

1 See also [19,29,27] for other work on D-branes in LG theories. 


\subsection{Sheaves on $\mathbb{P}^{n}$ and Beilinson's construction}

A broad class of CY threefolds arise as submanifolds of toric Fano manifolds. This is good in simple cases such as the quintic, but becomes cumbersome in general, when the codimension becomes high. Fortunately the class stays large enough even restricting to the case of hypersurfaces. This suggests that we understand sheaves on the CY as the restriction of those from the ambient space. This is standard procedure both in physics and mathematics. As we mentioned one can do it for more general toric ambient spaces, but here we just consider $\mathbb{P}^{4}$.

The simplest physical way to motivate the type of construction we use, is that we will choose $n$ generating branes (a "basis"), call them $S_{i}$ with $1 \leq i \leq n$. We then get all branes by forming all possible bound states of these. This is done by considering the world-volume theories of $N_{i}$ branes of type $S_{i}$; each BPS bound state will be a supersymmetric vacuum of at least one of these theories.

In the case of the quintic, one simple choice of basis is the line bundles $\mathcal{O}(n)$ for some sequence of five consecutive $n$. These span the $\mathrm{K}$ theory of $\mathbb{P}^{4}$, and an index 25 sublattice of the $\mathrm{K}$ theory of the quintic. An equally good basis, dual in a sense explained in [3, 12] is to take the exterior powers of the cotangent bundle,

$$
S_{n} \equiv \wedge^{5-n} \Omega_{\mathbb{P}^{4}}(n-5)[n-1], \quad 1 \leq n \leq 5 .
$$

We will denote the restriction of the $S_{n}$ to $\mathcal{M}$ as $B_{n}$. The [n-1] will be explained later; in particular the terms with $n-1$ odd are antibranes (with negative D6 charge).

Ideally, we would have a "universal" construction with the following property: not only can all branes be constructed, each brane can be constructed in only one way: this will mean that our world-volume theories precisely reproduce the moduli space of branes. This requires that the multiplicities $N_{i}$ be determined by the K theory class, so clearly this requires us to take exactly $n=\operatorname{dim} H^{\text {even }}$ generating branes.

However, even in simple examples, it turns out that to form all stable objects from such a basis, one must use both branes and the antibranes of the generating set. For this and many other reasons, one must work in a formalism which can describe general brane-antibrane bound states, while keeping track of all holomorphic information. Such a construction is the derived category of coherent sheaves.

There is a simple description of the derived category of coherent sheaves on $\mathbb{P}^{4}$ : it is the same as the derived category of quiver representations of a supersymmetric gauge theory given in [14] (and which we will review shortly). There are various mathematical and physical arguments that most, if not all, of these objects (restricted to the quintic) can appear as BPS branes at different points in Kähler moduli space. 


\subsection{Examples on $\mathbb{P}^{4}$}

We list here some simple examples which will reappear below. We will go through the translation from the geometry of sheaves to supersymmetric gauge theory in some detail in the first example.

The simplest example is a two term complex describing the bound state of two elementary branes, say

$$
\Omega(1) \stackrel{f}{\longrightarrow} \mathcal{O}
$$

The map $f$ is multiplication by a section of $(\Omega(1))^{*}=T(-1)$, the tangent bundle twisted by $O(-1)$. In physics notation, a section of $T(-1)$ is a tangent vector $f^{i} \partial_{i}$, specified by a set of functions $f^{i}$ (homogeneous of degree zero), modulo the "gauge symmetry" $f^{i} \sim f^{i}+z^{i} \epsilon$ for any $\epsilon$ of degree -1 . In mathematics, exactly the same idea is expressed by the Euler sequence

$$
0 \longrightarrow \mathcal{O}(-1) \longrightarrow \mathbb{C}^{5} \otimes \mathcal{O} \longrightarrow T(-1) \longrightarrow 0
$$

for which reason $T(-1)$ is often denoted by $\mathcal{Q}$ (for quotient).

Similarly, a section of $\Omega(1)$ is a (degree zero) one-form $\psi_{i} d z^{i}$ satisfying the constraint $z^{i} \psi_{i}=0$. The map $f$ in $(2.2)$ is then $\psi_{i} \longrightarrow f^{i} \psi_{i}$.

A holomorphic map of this type will have $f^{i}$ constant, so the space of these is $\operatorname{Hom}(\Omega(1), \mathcal{O}) \cong \mathbb{C}^{5}$. To realize this bound state of two elementary branes in the supersymmetric gauge theory requires five chiral multiplets $X^{i}$ with charge $(-1,+1)$ under $U(1) \times U(1)$; their vevs specify a map as $f^{i}=X^{i}$. The moduli space of supersymmetric vacua is the space of these vevs modulo the off-diagonal $U(1)$ gauge fixing and D-term constraint; if this constraint allows $X \neq 0$, the moduli space is $\mathbb{P}^{4}$.

The geometric interpretation of the bound state is the cohomology of the complex (2.2). Such an $f$ has a kernel but no cokernel, so the cohomology of (2.2) is a sheaf of rank

3. Call it $E_{1}$; we can express the construction with the exact sequence

$$
0 \longrightarrow E_{1} \longrightarrow \Omega(1) \stackrel{f}{\longrightarrow} \mathcal{O} \longrightarrow 0
$$

The sheaf $E_{1}$ is singular at the point $z^{i}=X^{i}$, so the moduli space is just the $\mathbb{P}^{4}$ parameterizing the choice of singular point.

In the derived category, this construction can be expressed as

$$
E_{1} \cong \underline{\Omega(1)} \longrightarrow \mathcal{O}
$$


As in [2], we underline the zero position in a complex.

Another simple example is

$$
\Omega^{4}(4) \stackrel{f}{\longrightarrow} \Omega^{3}(3) \stackrel{g}{\longrightarrow} \Omega^{2}(2)
$$

The two maps $f$ and $g$ are again both multiplications by sections of $T(-1)$, that is vectors in $\mathbb{C}^{5}$. But the condition that $(2.3)$ be a complex (physically, as we will review later, this is the superpotential constraint) has as unique solution $X_{f}^{i}=X_{g}^{i}$ up to rescaling. These rescaling do not give different sheaves (physically this is a gauge quotient), so these objects also have moduli space $\mathbb{P}^{4}$. This complex turns out to have its cohomology at the last node (as one might guess since this is the constituent of highest rank). It is another rank 3 sheaf, which we denote $E_{2}$. In the derived category, $E_{2}$ is equivalent to $\Omega^{4}(4) \longrightarrow \Omega^{3}(3) \longrightarrow \Omega^{2}(2)$.

One can continue in this vein, eventually constructing

$$
\Omega^{4}(4) \stackrel{X}{\longrightarrow} \Omega^{3}(3) \stackrel{X}{\longrightarrow} \Omega^{2}(2) \stackrel{X}{\longrightarrow} \Omega(1) \stackrel{X}{\longrightarrow} \mathcal{O}
$$

The constraints again force all the maps to be proportional, so all have been denoted by their vectors $X$, and the moduli space is again $\mathbb{P}^{4}$.

This sequence turns out to have cohomology only at the last term and this is in fact a Koszul resolution of a point on $\mathbb{P}^{4}$, dual to the one with line bundles $\mathcal{O}(k)$. We denote the structure sheaf of the point $z$ as $\mathcal{O}_{z}$.

Using these facts, and exact sequences of complexes, we can interpret the sheaves introduced so far as fitting into the distinguished triangle in the derived category

$$
E_{1} \longrightarrow \mathcal{O}_{z}[-1] \longrightarrow E_{2}[1] \longrightarrow E_{1}[1]
$$

So $E_{1}$ and $E_{2}$ are very similar, but differ by the addition of a point. Physically speaking, this implies that $E_{1}$ and $E_{2}$ differ by the addition of some 0-branes on $\mathbb{P}^{4}$.

Constructing more general sheaves will of course require taking some of the $N_{i}$ different from 0 or 1 . Here are three examples.

First, we can ask how the other more familiar objects on CY such as curves, hypersurfaces etc. look in this picture. Most of these will be intersections of objects of one dimension higher with the defining equation of the CY. Objects with nonzero codimension on $\mathbb{P}^{4}$ will be realized in the basis of line bundles by their Koszul resolutions; these can then be "dualized" to obtain an alternative Koszul resolution in the style of what we saw 
above for a point $z$. Alternatively we could simply plug the initial sheaf in the Beilinson machinery and obtain the same expression.

Consider the structure sheaf $\mathcal{O}_{P}$ of a hyperplane $P$ in $\mathbb{P}^{4}$. Slightly simpler for our purposes is $\mathcal{O}_{P}(1)$, its twist by $\mathcal{O}(1)$. In terms of line bundles this is

$$
\mathcal{O}_{P}(1) \cong \mathcal{O} \stackrel{f}{\longrightarrow} \underline{\mathcal{O}(1)} .
$$

In the Beilinson basis, it is

$$
\mathcal{O}_{P}(1) \cong \Omega(1) \stackrel{f}{\rightarrow} \underline{\mathcal{O} \otimes \mathbb{C}^{4}}
$$

Here $f$ is a $1 \times 4$ matrix of the the same maps used in (2.2); this leads to a moduli space of dimension $20-16=4$. By definition, this moduli space is the dual $\mathbb{P}^{4}$.

We can construct this moduli space in a more explicit way as follows. The map $f$ in (2.5) is again in the form $X^{i}$, but each component is now a matrix,

$$
\operatorname{Hom}\left(\mathcal{O}(1), \mathcal{O}_{P}(1)\right) \stackrel{X^{i}}{\longrightarrow} \operatorname{Hom}\left(\mathcal{O}, \mathcal{O}_{P}(1)\right)
$$

The generator of the first group is simply restriction on $P$; its image in the second group is simply $\left(e_{i}\right)_{\left.\right|_{P}}$, where $e_{i}$ is one of the basis elements of $\operatorname{Hom}(\mathcal{O}, \mathcal{O}(1))=H^{0}(\mathcal{O}(1))$. In particular, if for example $P$ is described by $z_{1}=0, X_{1}$ is the zero map (being its image $\left(e_{1}\right)_{\left.\right|_{P}}=0$. So we have 5 vectors in $\mathbb{C}^{4}$; or 4 vectors in $\mathbb{C}^{5}$. These 4 vectors span exactly the hyperplane, and we recover the moduli space as $\mathbb{P}^{4}$.

Subvarieties of higher codimension are constructed as longer complexes. For example, consider a surface $\Sigma$ in $\mathbb{P}^{4}$ defined as the intersection of two hyperplanes. One can easily match the $\mathrm{K}$ theory-class in the Beilinson basis, giving

$$
\Omega^{2}(2) \stackrel{f}{\longrightarrow} \Omega(1) \otimes \mathbb{C}^{3} \stackrel{g}{\longrightarrow} \mathcal{O} \otimes \mathbb{C}^{6} \longrightarrow \mathcal{O}_{\Sigma}(2)
$$

An instructive exercise is to compute the tensor product of the structure sheaves of two hyperplanes, $\mathcal{O}_{P}$ and $\mathcal{O}_{Q}$. Doing this in the line bundle basis leads to the Koszul complex; the complex above is dual to this.

As another example, one of the Recknagel-Schomerus states $|11000\rangle$ corresponds to

$$
\Omega^{2}(2) \stackrel{X}{\longrightarrow} \Omega(1) \otimes \mathbb{C}^{2} \stackrel{X}{\longrightarrow} \mathcal{O}
$$

From conformal field theory, it is easy to see that this state has 11 linearized moduli. 


\subsection{Restriction to a hypersurface}

We now consider a degree $d$ hypersurface $\mathcal{M}$ in $\mathbb{P}^{D+1}(\mathcal{M}$ is $\mathrm{CY}$ if $d=D+2)$, and explain why in complex dimension $D \geq 3$, sheaves on a hypersurface are very closely related to those on the ambient space, in distinction to the lower dimensional cases. We start with the question, can two different sheaves $E$ and $E^{\prime}$ restrict to the same sheaf on $\mathcal{M}$ ?

Let us start with the simplest case of restricting a bundle. Geometrically, one pictures a vector bundle as locally trivial over patches $\alpha$, related by transition functions $g_{\alpha \beta}$, with two different bundles equivalent if they are related by holomorphic gauge transformations $g_{\alpha \beta}^{\prime}=g_{\alpha} g_{\alpha \beta} g_{\beta}^{-1}$. In this language, the question is whether allowing gauge transformations $g_{\alpha}$ which can become singular off $\mathcal{M}$ leads to additional equivalences between bundles.

This question can be rephrased more mathematically as follows: does there exist an isomorphism between $E$ and $E^{\prime}$ on $\mathcal{M}$, i.e. an invertible $\operatorname{Hom}\left(E, E^{\prime}\right)$, which is not just the restriction of a $\operatorname{Hom}\left(E, E^{\prime}\right)$ between the bundles on the ambient space?

There is an exact sequence which is relevant for this question, which relates the cohomology of bundles on $\mathcal{M}$ to that on the ambient space. It can be derived by tensoring the bundles on the ambient space with the exact sequence

$$
0 \longrightarrow \mathcal{O}_{\mathbb{P}^{D+1}}(-d) \longrightarrow \mathcal{O}_{\mathbb{P}^{D+1}} \longrightarrow \mathcal{O}_{\mathcal{M}} \longrightarrow 0
$$

We obtain

$$
\begin{aligned}
& 0 \longrightarrow \operatorname{Hom}\left(E, E^{\prime}(-d)\right) \longrightarrow \operatorname{Hom}\left(E, E^{\prime}\right) \longrightarrow \operatorname{Hom}\left(E,\left.E^{\prime}\right|_{\mathcal{M}}\right) \longrightarrow \\
& \operatorname{Ext}^{1}\left(E, E^{\prime}(-d)\right) \longrightarrow \operatorname{Ext}^{1}\left(E, E^{\prime}\right) \longrightarrow \operatorname{Ext}^{1}\left(E,\left.E^{\prime}\right|_{\mathcal{M}}\right) \longrightarrow \\
& \cdots \\
& \operatorname{Ext}^{D+1}\left(E, E^{\prime}(-d)\right) \longrightarrow \operatorname{Ext}^{D+1}\left(E, E^{\prime}\right) \longrightarrow \operatorname{Ext}^{D+1}\left(E,\left.E^{\prime}\right|_{\mathcal{M}}\right) \longrightarrow 0
\end{aligned}
$$

Here Hom $\equiv \operatorname{Hom}_{\mathbb{P}^{D+1}}$ and Ext $\equiv \operatorname{Ext}_{\mathbb{P}^{D+1}}$ are on $\mathbb{P}^{D+1}$. The last groups in each row can be rewritten using the following basic fact [25]: $\operatorname{Ext}_{M}^{i}(E, \mathcal{E})=H^{i}\left(M, E^{*} \otimes \mathcal{E}\right)$ whenever $E$ is a bundle on $M$ and $\mathcal{E}$ whatever sheaf of $\mathcal{O}$-modules. In our case we have

$$
\begin{aligned}
\operatorname{Ext}^{i}\left(E,\left.E^{\prime}\right|_{\mathcal{M}}\right) \cong H^{i}\left(\mathbb{P}^{D+1},\left.E^{*} \otimes E^{\prime}\right|_{\mathcal{M}}\right) & \cong H^{i}\left(\mathbb{P}^{D+1},\left.\left(E^{*} \otimes E^{\prime}\right)\right|_{\mathcal{M}}\right) \\
& \cong H^{i}\left(\mathcal{M},\left.\left(E^{*} \otimes E^{\prime}\right)\right|_{\mathcal{M}}\right) \cong \operatorname{Ext}_{\mathcal{M}}^{i}\left(\left.E\right|_{\mathcal{M}},\left.E^{\prime}\right|_{\mathcal{M}}\right) .
\end{aligned}
$$

We also have Serre duality on $\mathbb{P}^{D+1}$, which states that

$$
\operatorname{Ext}^{D+1-n}\left(E, E^{\prime}\right) \cong \operatorname{Ext}^{n}\left(E^{\prime}, E(-D-2)\right)^{*}
$$


and allows rewriting first groups in each row. Summing up, we can rewrite (2.7) as

$$
\begin{aligned}
& 0 \longrightarrow \operatorname{Ext}^{D+1}\left(E^{\prime}, E(d-D-2)\right) \longrightarrow \operatorname{Hom}\left(E, E^{\prime}\right) \longrightarrow \operatorname{Hom}_{\mathcal{M}}\left(\left.\left.E\right|_{\mathcal{M}} E^{\prime}\right|_{\mathcal{M}}\right) \longrightarrow \\
& \operatorname{Ext}^{D}\left(E^{\prime}, E(d-D-2)\right) \longrightarrow \operatorname{Ext}^{1}\left(E, E^{\prime}\right) \longrightarrow \operatorname{Ext}_{\mathcal{M}}^{1}\left(\left.E\right|_{\mathcal{M}},\left.E^{\prime}\right|_{\mathcal{M}}\right) \longrightarrow \\
& \quad \cdots \\
& \operatorname{Hom}\left(E^{\prime}, E(d-D-2)\right) \longrightarrow \operatorname{Ext}^{D+1}\left(E, E^{\prime}\right) \longrightarrow 0 \longrightarrow 0
\end{aligned}
$$

From this we can see that any $\operatorname{Ext}_{\mathcal{M}}^{i}$ will receive contributions from both $\operatorname{Ext}_{\mathbb{P}^{D+1}}^{i}$ and a $\operatorname{Ext}_{\mathbb{P}^{D+1}}^{D-i}$ twisted by $d-D-2.2$ ) For the case of $\mathcal{M}$ a $\mathrm{CY}$ (i.e. $d=D+2$ ), we see that extra $\operatorname{Ext}^{i}\left(E, E^{\prime}\right)$ will come from $\operatorname{Ext}^{D-i}\left(E^{\prime}, E\right)$ on $\mathbb{P}^{D+1}$, or $H^{D-i}\left(M, E^{\prime *} \otimes E\right)$ if $E^{\prime}$ is a bundle.

Now for $[E]=\left[E^{\prime}\right]$ (both have the same $\mathrm{K}$ theory class), $c_{1}\left(E^{\prime *} \otimes E\right)=0$. For such bundles on a Fano variety, typically the higher cohomology groups vanish for sufficiently high degree, as formalized in vanishing theorems [24]. We will make this more precise below in the case of most interest to us. In general, it is easy to come up with examples with nonzero $H^{1}$ (any object with moduli), and examples are known with nonzero $H^{2}$ (any object with obstructed deformations), but already $H^{3}$ is not typical.

In $D=1$, this means that one always gains extra linearized moduli (elements of $\left.\operatorname{Ext}^{1}(E, E)\right)$ upon restriction, and typically gains extra elements of $\operatorname{Hom}\left(E, E^{\prime}\right)$ as well. This leads to drastic differences between bundles on the ambient space and the hypersurface.

In $D=2$, every $\operatorname{Ext}^{1}(E, E)$ on $\mathbb{P}^{3}$ will lead to a Serre dual $\operatorname{Ext}^{1}(E, E)$ on K3 by this argument. This type of pairing is required by general considerations (we know that moduli spaces of bundles on K3 are hyperkähler) but again complicates the story.

In $D \geq 3$, the new morphisms required by Serre duality on $\mathcal{M}$ are higher cohomology, so they do not affect the story as directly. The pairing $\operatorname{Ext}^{1} \cong \operatorname{Ext}^{2 *}$ in $D=3$ is quite important as it is responsible for the fact that the space of holomorphic objects can be described by a superpotential, as we discuss below.

2 A similar story can be told about the more general case in which we start from a Fano variety and a section of the anticanonical bundle. This also works well if this bundle is ample, which will be the case if the original weighted projective space did not have singularities intersecting the hypersurface. On the other hand, ampleness can be violated if we have to resolve singularities which intersect the hypersurface. A simple example is a hypersurface in $\mathbf{W P}_{1,1,1,3,3}$. Physically, the rational boundary states in these Gepner models do not span the K theory. 


\subsection{Quiver gauge theory and spherical bundles}

The mathematical considerations of the previous subsection have their most direct physical translation in the quiver gauge theories of B-type branes. The relevant quiver gauge theory on $\mathbb{P}^{4}$, or more precisely the quiver gauge theory for the non-compact orbifold $\mathrm{CY}, \mathbb{C}^{5} / \mathbb{Z}_{5}$, was written down in 12. The nodes of the quiver correspond to the basis branes $S_{i}$, and links to matter fields $\operatorname{Ext}^{p}\left(S_{i}, S_{j}\right)$.

We review the LG orbifold derivation of the quiver theory in section 4, but here summarize the result. The spectrum contains all odd $p$-forms on $\mathbb{C}^{5}$, i.e. $p=1,3,5$, and the orbifold projection enforces $j-i=p(\bmod 5)$. We denote the corresponding bifundamental matter as $X^{i}$ with $1 \leq i \leq 5$ (for $p=1$ ), and $Y^{i j k}$ (for $p=3$; we also write $\left.Y_{i j}=\epsilon_{i j k l m} Y^{k l m}\right)$. There is also a $p=5$-form $Z^{i j k l m}$ whose bosonic component has string-scale mass and which will not play a role until later, so we drop it here. The resulting quiver with $X$ arrows in groups of five and $Y$ arrows in groups of ten is

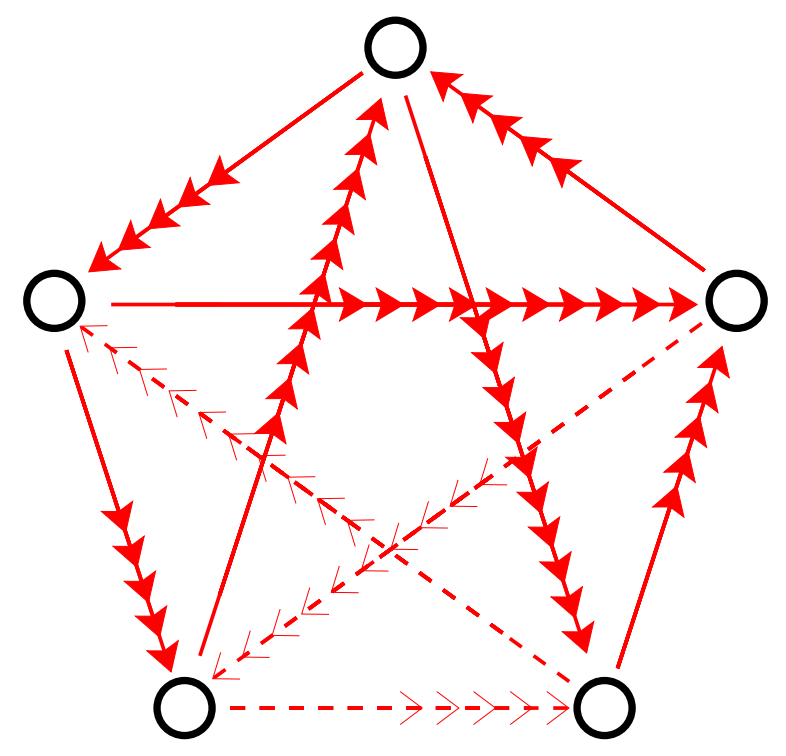

Figure 1. The quintic quiver.

Following the McKay correspondence, we make the identification (2.1). As explained in [14], one should include "flow of gradings" $[n-1]$ in comparing these objects to the large volume limit. The simplest argument for this is that the large volume interpretation of the fields $X^{i}$ is as the holomorphic maps $\operatorname{Hom}\left(S_{i}, S_{i+1}\right)$ (exactly the ones which appeared in (2.2)), which between branes and antibranes lead to tachyonic matter multiplets. However, at the orbifold point, the $S_{i}$ are all "branes" and the $p=1$ matter $X^{i}$ is massless. These two assignments of gradings are related precisely by the flow $S_{i} \rightarrow S_{i}[i+1]$. Similarly 
the $Y^{[i j k]}$ come from $\operatorname{Hom}\left(S_{i}, S_{i+3}\right)$. Note that the solid lines and dotted lines in figure 1 behave differently in the large volume limit; the dotted lines are obtained by flow from the Serre duals of the maps above.

We also have the cubic superpotential

$$
\mathcal{W}=X^{i} X^{j} Y_{i j}
$$

We will review the LG computation of this in section 4. It can also be understood as a five-form analog of the holomorphic Chern-Simons action.

Now, solutions of $\mathcal{W}^{\prime}=0$ (mod complex gauge equivalence) are exactly objects which can be used in a Beilinson construction of the derived category of sheaves on $\mathbb{C}^{5} / \mathbb{Z}_{5}$, in direct analogy to the familiar case of $\mathbb{C}^{3} / \mathbb{Z}_{3}$. All moduli of these sheaves are explicitly present; in gauge theory terms, as allowed variations of the fields $X$ and $Y$. In mathematical terms, these are the general deformations of a complex, meaning anything with total degree $1, H^{j}\left(\mathcal{M}, S_{i} \otimes S_{i+k}\right), j+k=1$; some of this is described in [14] and in appendix A.

We now want to study the restriction of this entire category to the quintic hypersurface. We start by restricting the basis branes, $B_{i}=\left.S_{i}\right|_{\mathcal{M}}$. Since the $S_{i}$ have no higher cohomology $\operatorname{Ext}^{p}(E, E)$ for any $p \geq 1$, one can see using the long exact sequence (2.8) that all of these restrict to "spherical bundles" on $\mathcal{M}$, i.e. bundles with $\operatorname{Hom}(E, E)=\operatorname{Ext}^{D}(E, E)=\mathbb{C}$ and no other cohomology.

Thus the nodes of the quiver gauge theory on $\mathbb{P}^{4}$ acquire no new adjoint matter on restriction. Similarly, one can check that the matter content $\operatorname{Ext}^{p}\left(B_{i}, B_{j}\right)$ agrees with the $\mathbb{C}^{5} / \mathbb{Z}_{5}$ quiver, but with different values of $p$ in the cases where Serre duality was involved, because one is now doing this in three dimensions. The flow of gradings also works in a similar way, but now with shifts $[3(n-1) / 5]$ instead of $[n-1]$, as shown in [14].

So far making the restriction has been rather trivial, supporting the rather optimistic claims of the previous subsection. However, the most obvious sign that not everything can be this simple is that the $\mathrm{K}$ theory of the quintic is a rank 4 group, while we have five distinct elementary branes $B_{i}$. This is the appropriate number for $\mathbb{P}^{4}$, but it is clear that a given $\mathrm{K}$ theory class on the quintic can be represented as a bound state in this basis in more than one way. The basic example of this is the point on $\mathbb{P}^{4}$, which realizes the fourth Chern class $c_{4}$. A generic point on $\mathbb{P}^{4}$, not lying on the hypersurface, restricts to nothing on the hypersurface, consistent with losing this Chern class.

3 A point on the hypersurface restricts to a two-term complex $O_{p t} \stackrel{0}{\longrightarrow} O_{p t}$, a direct sum of the $D 0$ and $\bar{D} 0$. This is unstable and physically would annihilate to the vacuum, but as a holomorphic object it is distinct from the null object. 
Thus, there must be equivalences between configurations which naively are distinct. We can get a simple example by considering the distinguished triangle (2.4). 1 . Since a generic point on $\mathbb{P}^{4}$ restricts to nothing, this directly implies that

$$
\left.\left.\left(E_{1}\right)\right|_{\mathcal{M}} \cong\left(E_{2}\right)\right|_{\mathcal{M}}
$$

Now this equivalence holds within the derived category of the quiver theory, and seeing it directly within supersymmetric gauge theory might not be easy (it is similar to the problem of describing brane-antibrane annihilation). However, a simple prediction that can be checked is that $E_{1}$ and $E_{2}$ should come in the same moduli spaces. On the other hand, according to quiver theory with the cubic superpotential, $E_{2}$ has more than 4 moduli on $\mathcal{M}$, thanks to the extra fields $Y$.

Evidently this quiver description of bundles on CY is flawed.

\subsection{Higher order superpotential terms from holomorphic Chern-Simons}

It does not take very much searching to find the flaw. From a physical point of view, there is no reason to think the cubic superpotential we postulated for $\mathbb{P}^{4}$ is correct for branes on $\mathcal{M}$; it could have higher order corrections. We will discuss the linear sigma model computation of these corrections below.

These higher order corrections can be understood geometrically as well. In this language, the superpotential is essentially the holomorphic Chern-Simons action

$$
S=\int \Omega \wedge\left(\frac{1}{2} A \bar{\partial} A+\frac{1}{3} A \wedge A \wedge A\right) .
$$

Naively, this action is cubic in variations of the gauge field. However, if one studies higher order deformations, one must do perturbation theory, or its equivalent. To express the superpotential in terms of linearized deformations, one must integrate out massive fields, which are not holomorphic, and this will produce higher order terms in the superpotential.

This phenomenon was described in [48] and was previously worked out in a more abstract context by various mathematicians; in the present context it seems to appear first in Merkulov [37]. It is also discussed in [39, 46, 32, 31].

4 This example includes as a special case the equivalence between the Recknagel-Schomerus boundary states $|10000\rangle$ and $|20000\rangle$ following from CFT field identifications. We thank J. Walcher for this comment. 
Let us now see more explicitly how these corrections arise from this point of view. We can describe these corrections as arising from Feynman diagrams [32, 46] of the holomorphic Chern-Simons theory (2.10). Every boundary operator, including every Ext ${ }^{1}$ corresponding to a linearized deformation, is an external leg. The vertex is the exterior product on forms with the tensor product of bundle factors. Finally, the propagators are the inverses of $\bar{\partial}$ operators of the appropriate bundles. (These inverses can be defined more properly using Hodge theory as $\bar{\partial}^{-1}=G \bar{\partial}^{\dagger}$; zero modes do not propagate.) So the cubic term $X^{i} X^{j} Y_{i j}$ we referred to earlier comes trivially from the vertex

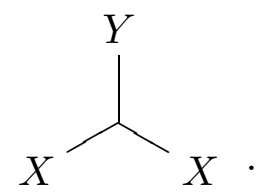

Figure 2. The cubic contribution to the superpotential.

In general, higher point functions involve a sum over planar tree diagrams of $\phi^{3}$ field theory. Mathematically, this has an interpretation in terms of $A_{\infty}$ algebras and categories.

Let us restrict attention to a quiver with three nodes, which can describe the object $E_{2}$. In this case, fortunately, there is only a single diagram. To see this, start by noting that only operator orderings which lead to gauge-invariant world-volume couplings can be non-zero; thus the leading correction will take the form $\operatorname{Tr} Y X X Y X X$. These operators correspond to zero-forms $X^{i}$, and three-forms (more precisely $(0,3)$ forms) $Y_{i j}$. Now we cannot have a vertex with $Y$ replaced by a propagator: this would be $\bar{\partial}^{-1}(X X)$, and we don't have any $(-1)$-form. We can instead write the two-form $\omega_{2} \equiv \bar{\partial}^{-1}(Y X)$, the oneform $\omega_{1} \equiv \bar{\partial}^{-1}\left(X \bar{\partial}^{-1}(Y X)\right)$, or a function $\omega_{0}$. If we want to make a $(0,3)$ form which then we can integrate against $\Omega$, the only options are $\omega_{3} X Y$, or $\omega_{2} \omega_{1}$. These two choices are equivalent by integration by parts:

$$
\int \Omega Y X \bar{\partial}^{-1}\left(X \bar{\partial}^{-1}(X Y)\right)=\int \Omega \bar{\partial} \bar{\partial}^{-1}(Y X) \bar{\partial}^{-1}\left(X \bar{\partial}^{-1}(X Y)\right)=-\int \Omega \bar{\partial}^{-1}(Y X) X \bar{\partial}^{-1}(Y X) .
$$

The final result for this term in the superpotential corresponds to the planar Feynman diagram in figure 3. Proceeding further with this computation would require us to have a concrete representation of $\bar{\partial}^{-1}=G \bar{\partial}^{\dagger}$. We do not, and instead we will compute this term in section 4 with topological field theory and linear sigma model methods.

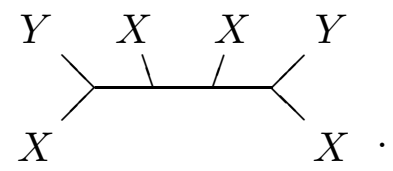

Figure 3. The sextic contribution. 


\subsection{Higher order superpotential terms resolve the problem}

Although the expression we just derived is not too explicit, it turns out to be good enough, in the sense that with some further physical input we can write a unique corresponding term in the superpotential.

The computation we described leads to a term

$$
I_{j_{1} j_{2} j_{3} j_{4} j_{5} j_{6} k_{1} k_{2} k_{3} k_{4}}^{(6)} \operatorname{Tr} Y^{j_{1} j_{2} j_{3}} X^{k_{1}} X^{k_{2}} Y^{j_{4} j_{5} j_{6}} X^{k_{3}} X^{k_{4}}
$$

where $I^{(6)}$ is some tenth order tensor. This tensor is constrained by symmetry; for example it is antisymmetric in $j_{1} j_{2} j_{3}$, and so forth.

Let us write an example of a tensor with the correct symmetries:

$$
I_{j_{1} j_{2} j_{3} j_{4} j_{5} j_{6} k_{1} k_{2} k_{3} k_{4}}^{(6)}=\epsilon_{j_{1} j_{2} j_{3} j_{4} j_{5}} c_{j_{6} k_{1} k_{2} k_{3} k_{4}}
$$

where $c_{i j k l m}$ is the totally symmetric tensor defining the polynomial which describes the quintic, $c_{i j k l m} z^{i} z^{j} z^{k} z^{l} z^{m}=0$.

Of course there are many other ways we could have contracted the indices. However, if we are interested in solutions of $W^{\prime}=0$, it suffices to know the sixth order term up to corrections proportional to $W^{\prime}$ at the lower order. In other words, we can assume the relations

$$
X^{i} X^{j}=X^{j} X^{i}
$$

and

$$
X^{i} Y_{i j}=Y_{i j} X^{j}=0
$$

This allows fairly general reorderings of the indices, and one can show that all of the possible invariants constructed from the two tensors $\epsilon$ and $c$ are equivalent up to these relations.

From the point of view of deformations, we must start with initial data satisfying the first relation, and the second relation survives the deformation. We discuss this further in the next section.

Let us check that this term resolves our contradiction, while not spoiling other good results. We start from the example of $E_{1}$ and $E_{2}$ from above, that initially prompted our discussion. We start with a solution for the $E_{2}$ quiver theory with $Y=0$. This requires all $X$ equal; by global symmetry we can take them to be $X^{i}=\delta^{i, 1}$. We should then consider 
hypersurfaces which do not intersect the point $z^{i}=X^{i}$ (otherwise $O_{z}$ will not restrict to zero and in fact $E_{1} \neq E_{2}$ ). This requires $c_{11111} \neq 0$.

With the additional superpotential term, the equations $W^{\prime}=0$, linearized about this configuration as we saw above, become explicitly

$$
\begin{aligned}
& 0=\epsilon_{i j k l 1} y^{i j k} \quad\left(X \cdot y=0=y \cdot X^{\prime}\right) \\
& \begin{array}{ccc}
0=x^{i}+c_{1111 j} y^{i j 1} & \forall i \neq 1 \\
0=c_{1111 k} y^{i j k} & \forall i, j \neq 1
\end{array} \quad\left(x^{\prime} \wedge X+X^{\prime} \wedge x+X^{2} X^{\prime 2} y=0\right)
\end{aligned}
$$

(remember that lower case letters denote candidate deformations; we drop the subscript 0 on initial-point values; the quintic term is now given by the superpotential we just computed). The first equation sets to zero all components of $y^{i j k}$ with $i, j, k \neq 1$; this leaves us with only the $y_{1 j k}, j, k \neq 1$, which are our 6 candidate moduli. The third equation sets all of the other components to zero, if $c_{11111} \neq 0$ : this means that there are no deformations arising from the $y_{i j k}$.

Thus both objects have the same moduli space and the contradiction is eliminated. The basic lesson is that the effect of restriction is in general non-trivial, but can be summarized in the higher order terms of the world-volume superpotential. We will illustrate this with more examples in section 5 .

\section{Restriction using spectral sequences}

An alternate approach to studying the restriction of sheaves is to use a spectral sequence, as we discuss in this section. This is not really required for the rest of the discussion, but is also instructive.

The phenomena we saw above would be visible already in the linearized deformations, if we can find these around a general complex. In this special case, one can get the correct deformation theory as a cohomology problem, but now using a cohomology which includes the nonholomorphic maps. The potential relevance of this cohomology was suggested by Diaconescu [11].

In terms of topological open string theory, what we will do now amounts to defining $Q_{E, F}=\bar{\partial}+Q_{E}-Q_{F}$ and allow for possible non-holomorphic maps; in other words we will consider a $n$-form $\psi^{(n)}$ to be $Q$ - exact if it obeys an equation like $Q \psi^{(n)}=\bar{\partial} \psi^{(n-1)}$. This looks complicated, and it is, but the complications can be reduced by appealing to the machinery of spectral sequences. 
We begin with the special case of a complex

$$
C \equiv\left\{B_{1} \stackrel{a_{1}}{\longrightarrow} B_{2} \stackrel{a_{2}}{\longrightarrow} B_{3}\right\}
$$

Starting with this complex, we proceed to construct a double complex. This is needed because deformations of a single holomorphic bundle $B$ are computed from $H^{1}$ of a complex, for example the Dolbeault one:

$$
\Omega^{0,0}(\mathcal{M}, B) \longrightarrow \Omega^{0,1}(\mathcal{M}, B) \longrightarrow \ldots
$$

where $\Omega^{p, q}(\mathcal{M}, B)$ are forms of type $(p, q)$ with values in the bundle $B$, and the maps in the complex are the Dolbeault differentials $\bar{\partial}_{B}$. The first cohomology of this complex $H_{\bar{\partial}}^{0,1}(\mathcal{M}, B)$ can be indeed reexpressed by Dolbeault theorem in the more familiar form $H^{1}(\mathcal{M}, B)$. The fact that we are dealing with a complex of bundles gives us another "direction" for the complex computing deformations. More precisely, we have to write down the double complex $E_{p, q}^{0}$ whose $p$-th line $(p=0, \ldots, 3)$ is, for our three-term example

$$
\begin{aligned}
\Omega^{0, p}\left(\mathcal{M}, B_{3}^{*} \otimes B_{1}\right) \stackrel{\tilde{d}^{\prime}}{\longrightarrow} & \Omega^{0, p}\left(\mathcal{M}, \oplus_{i=1}^{2} B_{i+1}^{*} \otimes B_{i}\right) \stackrel{\tilde{d}}{\longrightarrow} \Omega^{0, p}\left(\mathcal{M}, \oplus_{i=1}^{3} B_{i}^{*} \otimes B_{i}\right) \\
& \stackrel{d}{\longrightarrow} \Omega^{0, p}\left(\mathcal{M}, \oplus_{i=1}^{2} B_{i}^{*} \otimes B_{i+1}\right) \stackrel{d^{\prime}}{\longrightarrow} \Omega^{0, p}\left(\mathcal{M}, B_{1}^{*} \otimes B_{3}\right) .
\end{aligned}
$$

The maps in these lines are defined in a way which is similar to the complex one uses to compute Hom and Ext for a quiver without relations. For our three-term example, for instance, first map $\tilde{d}^{\prime}$ in $(3.3)$ is $f \mapsto\left(f \circ a_{2},-a_{1} \circ f\right)$. Tildes are there to emphasize how these maps are duals among them; see later. Names of these maps will occasionally mean both the maps between the bundles and, as in this case, the maps on the bundle-valued forms that they induce. First cohomology group of the double complex defined in this way gives then deformations of the original complex (3.1).

To compute the cohomology of a double complex (or at least a good approximation to it: we will come back to this later), the tool which is usually effective is a spectral sequence. For a general introduction, see for instance [5, 24, 36]: here we will concretely follow the procedure and explain it. Spectral sequence procedure computes for us a series of approximations to the sought-for cohomology. First approximation we choose to be the cohomology of the vertical arrows. This means that we can replace each column (which 
has the form of (3.2)) with its cohomology. This gives us a new double complex, whose $p$-th line is now

$$
\begin{aligned}
& H^{0, p}\left(\mathcal{M}, B_{3}^{*} \otimes B_{1}\right) \longrightarrow H^{0, p}\left(\mathcal{M}, \oplus_{i=1}^{2} B_{i+1}^{*} \otimes B_{i}\right) \longrightarrow H^{0, p}\left(\mathcal{M}, \oplus_{i=1}^{3} B_{i}^{*} \otimes B_{i}\right) \\
& \longrightarrow H^{0, p}\left(\mathcal{M}, \oplus_{i=1}^{2} B_{i}^{*} \otimes B_{i+1}\right) \longrightarrow H^{0, p}\left(\mathcal{M}, B_{1}^{*} \otimes B_{3}\right)
\end{aligned}
$$

where the horizontal maps are the maps induced on the cohomologies by the horizontal maps in (3.3). This is our first approximation to the cohomology of the double complex.

So far we never specified what the $B_{i}$ were; we now choose them to be $\left.S_{i}\right|_{\mathcal{M}}=$ $\left.\left(\Omega^{5-i}(5-i)\right)\right|_{\mathcal{M}}$ as in (2.1). Then the cohomology groups in (3.4) are the $\operatorname{Ext}_{\mathcal{M}}^{p}$ of the $B_{i}=\left.\left(S_{i}\right)\right|_{\mathcal{M}}$ and we can compute them from the $\operatorname{Ext}_{\mathbb{P}^{4}}^{p}\left(S_{i}, S_{j}\right)$ again using (2.8). The result is that the only nonzero groups are

$$
\operatorname{Hom}_{\mathcal{M}}\left(B_{i}, B_{j}\right)=\operatorname{Hom}_{\mathbb{P}^{4}}\left(S_{i}, S_{j}\right)=\Lambda^{j-i} V=\operatorname{Ext}_{\mathcal{M}}^{3}\left(B_{j}, B_{i}\right), \quad i \leq j,
$$

where $V \equiv \mathbb{C}^{5}$. More generally we can say that an exceptional series on $F$ becomes a so-called spherical series of bundles on $\mathcal{M}$ [44], analogously to what stated above about a single bundle. The double complex is thus now

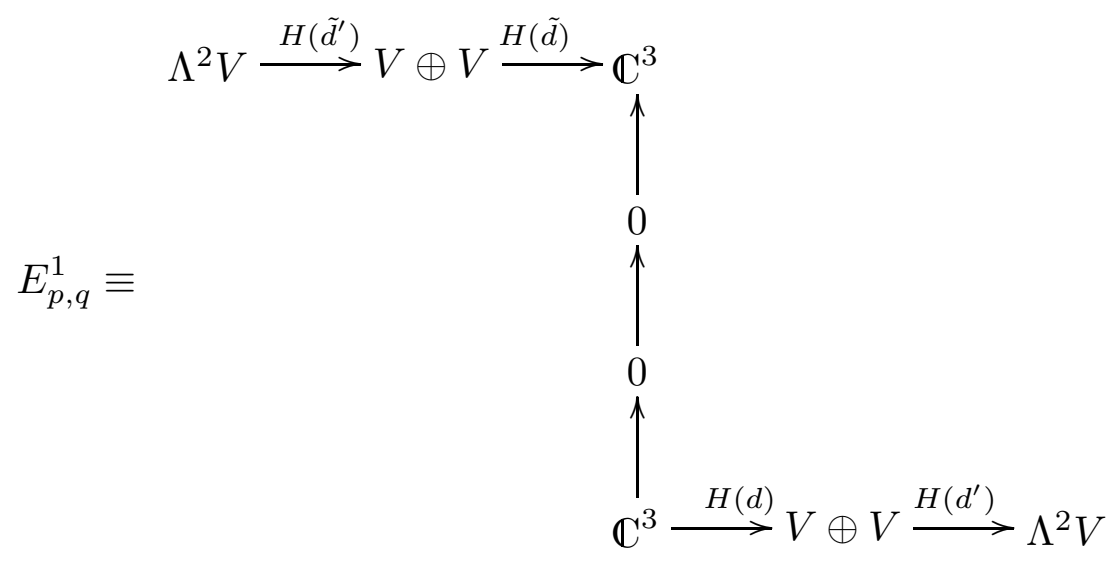

with zeroes and arrows going up and right to fill; $H$ (maps) is to remind that these arrows are induced on cohomologies by maps. The $(0,0)$ harmonic forms with values in $B_{1}^{*} \otimes B_{3}$, or by a little abuse of language their Serre duals in the upper left corner, $(0,3)$ harmonic forms with values in $B_{3}^{*} \otimes B_{1}$, are nothing but what we called earlier $y_{i j}$; the $(0,0)$ harmonic forms with values in $\left(B_{1}^{*} \otimes B_{2}\right) \oplus\left(B_{2}^{*} \otimes B_{3}\right)$ (or again their duals) are what we called collectively earlier $X^{i}$, and that we now call more precisely $\left(x^{i}, x^{i}\right)$. We use lower-case letters here to distinguish these deformations from the initial-point value of the fields, as we shall see shortly. 
Second approximation is now to compute the cohomology of the horizontal maps in (3.5). 0-th line is more explicitly

$$
\begin{array}{ccc}
\mathbb{C}^{3} & \longrightarrow \quad V \oplus V & \longrightarrow \Lambda^{2} V \\
(\alpha, \beta, \gamma) & \left.\mapsto(\alpha-\beta) X_{0},(\beta-\gamma) X_{0}^{\prime}\right) & \\
\left(v, v^{\prime}\right) & \mapsto\left(X_{0}^{\prime} \wedge v+v^{\prime} \wedge X_{0}\right),
\end{array}
$$

where with hindsight we call $X_{0}, X_{0}^{\prime}$ the global sections of $\operatorname{Hom}\left(B_{1}, B_{2}\right), \operatorname{Hom}\left(B_{2}, B_{3}\right)$ which represent the maps in the original complex $C$. It is easy to see that cohomology of (3.6) is $(1,4,6)$. Third line is but the dual complex to this, and as a consequence its cohomology is $(6,4,1)$. We can now display our second approximation to the cohomology of the double complex as

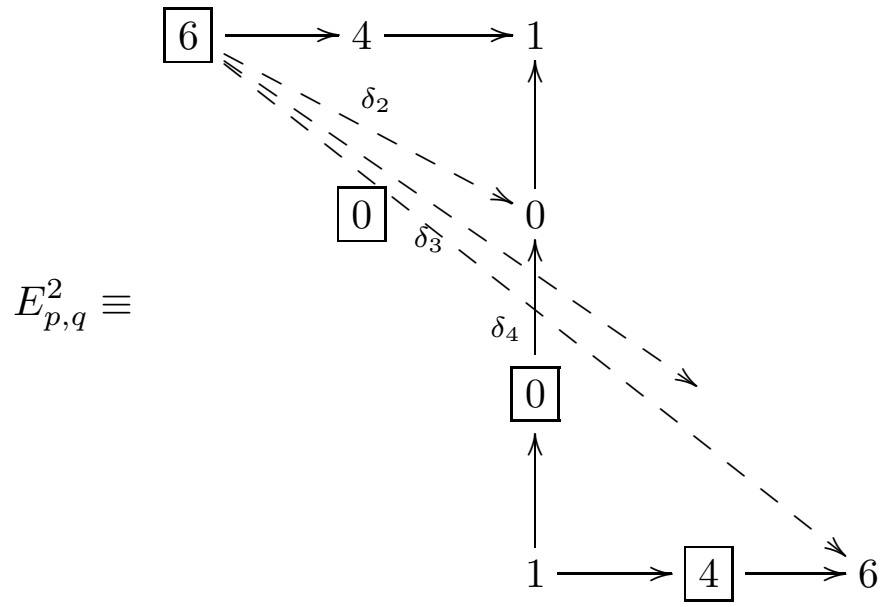

Third approximation is to compute the cohomology of a new sort $\delta_{2}$ of oblique arrows, going down 1 and right 2, as shown in (3.7) (the other two oblique arrows and the boxes are there for future reference). This is defined as follows. Take for example as source space the upper left corner of (3.7). These are the 6 harmonic three-forms $y$ with values in $B_{3}^{*} \otimes B_{1}$ 目 that are in the kernel of the horizontal map $H\left(\tilde{d}^{\prime}\right)$ - in particular they are not among the 4 three-forms with values in $B_{3}^{*} \otimes B_{2} \oplus B_{2}^{*} \otimes B_{1}$ that are displayed in (3.7); they are zero in this group. Being zero in this cohomology group means being $\bar{\partial}$ closed; we can thus find a two-form $\alpha$ with values in $B_{3}^{*} \otimes B_{2} \oplus B_{2}^{*} \otimes B_{1}$, such that $\bar{\partial} \alpha=\tilde{d}^{\prime} y$. We define $\delta_{2} y$

5 Concretely, these can be expressed in terms of holomorphic sections $y_{[i j]}$ of $B_{1}^{*} \otimes B_{3}$ as $\Omega(y, \cdot)$, where $($,$) is a hermitian metric for the bundle: in components, \Omega y_{\left[i^{\prime} j^{\prime}\right]} h^{\left[i^{\prime} j^{\prime}\right][i j]}$. The sections $y_{[i j]}$ themselves can be got instead easily looking at the definitions of the bundles $B_{i}$ and at the Euler sequence. 
now as the result of applying next horizontal map: $\delta_{2} y \equiv \tilde{d} \alpha$. Summarizing, this is a map between the 6 elements in the upper left corner, call it position $(-2,3)$, and the elements in position $(0,2)$. As this cohomology group in $E^{2}$, (3.7), is zero, this map is trivially zero. Looking at (3.7) a little more one discovers that there actually any other arrow of the type $\delta_{2}$ (going right 2 and down 1 ) will be zero by this trick. So our next approximation, which would consist in replacing $E_{p, q}^{2}$ by its cohomology under the $\delta_{2}$ maps, is actually the same as $E^{2}$ again.

It would be wrong, however, to conclude that the approximation procedure has finally converged. Indeed, again, the image under $\delta_{2}$ is zero not as a form, but as an element in the cohomology $E^{2}$. This means that we can go on with our descent procedure:

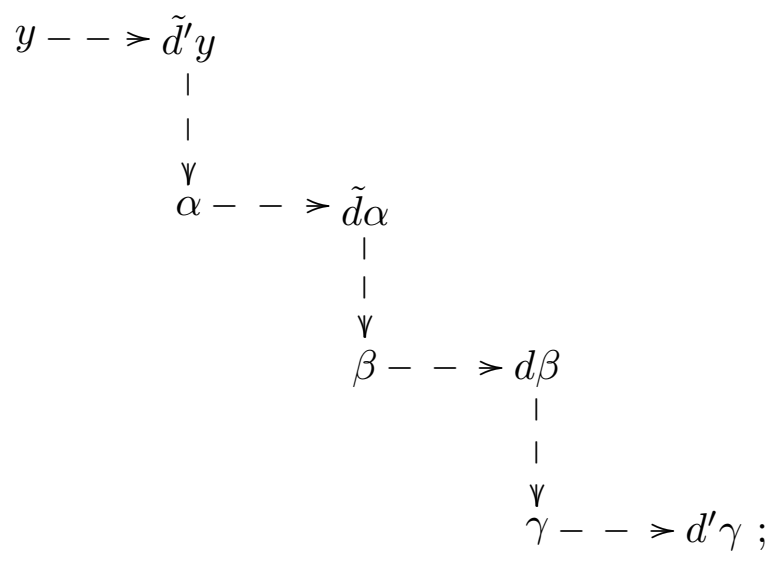

by definition, $\bar{\partial} \beta=\tilde{d} \alpha$, and $\delta_{3} y \equiv d \beta$. Since again this ends on a zero on $E^{3}=E^{2}$, then we can go on and define by $\bar{\partial} \gamma=d \beta$ the map $\delta_{4} y=d^{\prime} \gamma$. Compare now (3.7): this does not end now on a zero, but again on 6 . So it is potentially non vanishing. The example for $\delta_{4}$ was cleverly chosen: this is the only arrow having a chance to be nonzero. It is moreover relevant to our problem: in (3.7) we have boxed the cohomology groups which can contribute to moduli (they are in position $(p, q)$ such that $p+q=1$, and we have recalled earlier deformations are in first total cohomology group). From this point on, arrows will be always too long and will therefore always be zero: so our approximation $E_{p, q}^{5}$ (which is essentially nothing but modifying $E^{3}$ by computing the cohomology of the map $\delta_{4}$ ) will be the final one, $E^{5}=E^{\infty}$ 国. At the end of the day, the number of our moduli

6 In general even this final approximation is not exactly what one wants: The direct sum of the groups along the boxed diagonal in $E^{5}$ would not be exactly the first cohomology group of the original double complex (3.3). Working with vector spaces we will however see no difference. Note that for $B_{i}$ spherical bundles as in this case, things can be anyway made more precise building an exact sequence, similar to the Gysin sequence for sphere bundles, summarizing the content of the spectral sequence. 
is $4+\operatorname{ker}\left(\delta_{4}\right)$. Although we have not performed the convoluted computation, the lack of reasons to its vanishing at first steps and symmetry reason makes one suspect it is actually an isomorphism.

The map $\delta_{4}$ was evidently what was not taken in account in the preliminary analysis in subsection 2.3. To solve the apparent contradiction we had there, exemplified by the example (2.9), clearly it is $\delta_{4}$ that has to lift the extra fields, all the remaining $Y$ in that case. This should also agree with the other argument we gave, the presence of a further term in the superpotential. To look at this in more detail, let us reexpress our computation in a way more suitable to generalizations.

At the stage (3.5), we were faced with harmonic forms $x_{i}, x_{i}^{\prime}, y_{[i j]}$, following the notation introduced there. We can as well consider them as elements $x, x^{\prime}, y$ of $V \oplus V \oplus \Lambda^{2} V$ and express more significantly our next formulas in terms of usual exterior algebra, with wedges and contractions. Going to $E^{2}$ meant to compute the cohomology of the horizontal arrows. For $x, x^{\prime}$ this only means to consider the ones which satisfy (looking back at first map in (3.6) ) $x^{\prime} \wedge X_{0}+X_{0}^{\prime} \wedge x=0$, modulo $\left(x, x^{\prime}\right) \cong\left(x, x^{\prime}\right)+\left(X_{0} A_{1}-A_{2} X_{0}, X_{0}^{\prime} A_{2}-A_{3} X_{0}^{\prime}\right)$; in the example considered so far the $A_{i}$ are still numbers. As for the $y$, the condition of being in the cokernel can be equally well expressed as being in the kernel of the dual map $\tilde{d}^{\prime}$ : this means $X_{0} \cdot y=0=y \cdot X_{0}^{\prime}$ (in components, $\left.X_{0}^{i} y_{i j}=0=y_{i j} X_{0}^{\prime j}\right)$.

In the following steps there were no essential changes to this picture, but for the map $\delta_{4}$. We can see from the summarizing scheme (3.8) that it involves four horizontal maps, and each horizontal map is linear in $X_{0}, X_{0}^{\prime}$. In fact, coming back to the discussion around (3.8), one can reexpress the result as

$$
\delta_{4} y=d^{\prime} \bar{\partial}^{-1}\left(d \bar{\partial}^{-1}\left(\tilde{d} \bar{\partial}^{-1}\left(\tilde{d}^{\prime} y\right)\right)\right)
$$

since each of the $d, d^{\prime} \ldots$ is linear in $X_{0}$ and $X_{0}^{\prime}$, we see that this is nothing but a sum of Merkulov diagrams of the sextic form in figure 3! We can denote the ensuing relation symbolically ${ }^{\prime}$ as $\left(y X_{0}^{2} X_{0}^{\prime 2}\right)_{c}=0$. The subscript ${ }_{c}$ is to distinguish this from a slightly different object we will meet shortly.

When generalizing the three-term complex analyzed so far to general three-term complexes with general $n_{i}$ (not necessarily $n_{1}=n_{2}=n_{3}=1$ ), first change in previous discussion is that $x, x^{\prime}, y$ become now also matrices, gaining extra indices $\left(x_{a_{1} a_{2}}, x_{a_{2} a_{3}}^{\prime}, y_{a_{3} a_{1}}\right.$,

7 In the sense that we have not yet established the way in which the indices are contracted, and whether the whole thing is actually non-vanishing: the expression only shows how many of the various fields should appear. 
where $\left.a_{i}=1, \ldots, n_{i}\right)$ so that they are elements now of $V \otimes$ End $\left(n_{1}, n_{2}\right), V \otimes$ End $\left(n_{2}, n_{3}\right)$ and $\Lambda^{2} V \otimes$ End $\left(n_{3}, n_{1}\right)$ respectively. But apart from this, previous expressions were written in such a way that hold true in this more general case, with the only proviso that the products are now tensor products of (wedge and contractions in the exterior algebra of $V$ ) $\otimes$ (matrix multiplication). Summarizing the conditions, we have that our deformations are described by

$$
\begin{gathered}
\left(x, x^{\prime}, y\right) \quad \text { such that } \quad x^{\prime} \wedge X_{0}+X_{0}^{\prime} \wedge x=0, \quad X_{0} \cdot y=0=y \cdot X_{0}^{\prime} \quad\left(y X_{0}^{2} X_{0}^{\prime 2}\right)_{c}=0 \\
\text { and modulo }\left(x, x^{\prime}, y\right) \sim\left(x, x^{\prime}, y\right)+\left(X_{0} A_{1}-A_{2} X_{0}, X_{0}^{\prime} A_{2}-A_{3} X_{0}^{\prime}, 0\right) .
\end{gathered}
$$

We will see now that the same deformations, but for the quintic relation in (3.9), come from the quiver in figure 1 with cubic superpotential $\operatorname{tr}\left(Y_{[i j]} X_{i} X_{j}\right)$. Indeed, this gives us relations $X^{\prime} \wedge X=0=X \cdot Y=Y \cdot X^{\prime}$. Moreover, different representations are also considered to be equal if they go into each other by reparameterizations of the three vector spaces $\mathbb{C}^{n_{i}}$ : infinitesimally this yields $\left(X, X^{\prime}, Y\right) \sim\left(X_{0}, X_{0}^{\prime}, Y_{0}\right)+\left(X_{0} A_{1}-\right.$ $\left.A_{2} X_{0}, X_{0}^{\prime} A_{2}-A_{3} X_{0}^{\prime}, Y_{0} A_{1}-A_{2} Y_{0}\right)$. To analyze deformations, we use an apparently "naive" procedure which is actually nothing but a translation of the so-called standard complex (for categories) [38]; we sketch an account of it in appendix a. That is, we simply write the fields in the form of a "background value" plus infinitesimal deformations: $\left(X, X^{\prime}, Y\right)=$ $\left(X_{0}+x, X_{0}^{\prime}+x^{\prime}, y\right)$; of course this requires that $X_{0}^{\prime} \wedge X_{0}=0$. Inserting this into our relations, we find

$$
\left(X_{0}^{\prime} \wedge X_{0}\right)+X_{0}^{\prime} \wedge x+x^{\prime} \wedge X_{0}=0=X_{0} \cdot y=y \cdot X_{0}^{\prime}
$$

first piece is there only for illustration, and as we have said it vanishes. The identifications become likewise $\left(x, x^{\prime}, y\right) \sim\left(x, x^{\prime}, y\right)+\left(X_{0} A_{1}-A_{2} X_{0}, X_{0}^{\prime} A_{2}-A_{3} X_{0}, 0\right)$. Putting all together, we have exactly the same relations we had in (3.9) — but for the quintic relation at the end of first line.

We have, however, already seen in subsection 2.5 that a deformation of the superpotential is there; moreover this deformation seems to have exactly the same origin as the map $\delta_{4}$. It is a little subtler to see more precisely the agreement. Adding to the initial cubic superpotential tr $\left(Y_{[i j]} X_{i} X_{j}\right)$ a sextic term $Y^{2} X^{2} X^{\prime 2}$, we can see that for instance first relation becomes $X^{\prime} \wedge X+Y X^{2} X^{\prime 2}=0$, which infinitesimally gives

$$
X_{0}^{\prime} \wedge x+x^{\prime} \wedge X_{0}+y X_{0}^{2} X_{0}^{\prime 2}=0
$$


The quintic piece in (3.10) has been denoted differently from the one in (3.9), though we just argued they come from the same diagrams. Moreover, the two quintic objects appear differently: alone in (3.9), and summed to something else in (3.10). The reason for both things is as follows. The spectral sequence map $\delta_{4}$ takes values not exactly in the space of all the $y_{i j}$, but in its quotient by the image of the map $H\left(d^{\prime}\right)$, as we denoted in (3.5). So, once we have the brute result of the computation of the Merkulov diagram, which is what we denoted as $y X_{0}^{2} X_{0}^{\prime 2}$ in (3.9), imposing that this is zero means only, strictly speaking, that it is zero modulo the image of $H\left(d^{\prime}\right)$; that is, that this is zero modulo a term $X_{0}^{\prime} \wedge x+x^{\prime} \wedge X_{0}$, for some $x$ and $x^{\prime}$. A dual method to deal with the quotient space is to "fix the gauge invariance" and project to a subspace transverse to the orbits of the image of $H\left(d^{\prime}\right)$. The natural choice is to take exactly the same condition of transversality that the $y_{i j}$ satisfy, $X_{0}^{i} y_{i j}=0=y_{i j} X_{0}^{\prime j}$; this transversality fixes the gauge because the complexes in zeroth and third line of (3.7) are adjoint to each other, and kernel in one is dual to a cokernel (which is a quotient) in the other. So one has to subtract something to the "rough" $\left(y X_{0}^{2} X_{0}^{\prime 2}\right)$ in order to make it transverse to $X_{0}$ and $X_{0}^{\prime}, X_{0}^{i}\left(y X_{0}^{2} X_{0}^{\prime 2}\right)_{c i j}=0=\left(y X_{0}^{2} X_{0}^{\prime 2}\right)_{c i j} X_{0}^{\prime j}$. In general, this can be accomplished fixing metrics $\langle$,$\rangle on the spaces of the vector spaces of the X, X^{\prime}$ and $y$ and writing

$$
\delta_{4}(y)=\left(y X_{0}^{2} X_{0}^{\prime 2}\right)_{c}=\left(y X_{0}^{2} X_{0}^{\prime 2}\right)-\left\langle X^{\prime} \wedge\left(\left(y X_{0}^{2} X_{0}^{\prime 2}\right) \cdot X^{\prime}\right), \cdot\right\rangle-\left\langle\left(X \cdot\left(y X_{0}^{2} X_{0}^{\prime 2}\right)\right) \wedge X, \cdot\right\rangle
$$

This formula will become complete when we will write down what $\left(y X_{0}^{2} X_{0}^{\prime 2}\right)_{i j}$ is in next subsection; we will look then at examples to clarify this discussion.

As to the $x, x^{\prime}$, they are trivially never lifted in neither approach (once the relation $X_{0}^{\prime} \wedge x+x^{\prime} \wedge X_{0}=0$ is satisfied) : this is because such moduli are always trivially solutions of (3.10). Let us also note that, if we had been computing deformations of the same object but on $\mathbb{P}^{4}$, before restricting to $\mathcal{M}$, we would have had only zero-th line in (3.7); so the spectral sequence machinery would have stopped at that stage, and the only solutions we would have found would have been exactly these ones due to the $X$. So the $X$ are the moduli that were already present in $\mathbb{P}^{4}$, and the $Y$ are candidate new moduli. This reflects the splitting between these two kinds of moduli that we saw in the exact sequence (2.8). In fact, although we will not show this in detail here, one can see that the spectral sequence of this subsection leads exactly to the (2.8). This is because, for spectral sequences for which only two lines are nonvanishing, one can summarize the whole information of the spectral sequence in a more ordinary-looking exact sequence. This happens for instance 
when computing cohomology groups of sphere bundles; in that case the resulting exact sequence is called after Gysin [5]. In this case, exactly the same procedure would lead to (2.8) (note again that we called indeed our basic bundles $B_{i}$ spherical!); for $D=3$ there is indeed a map between $\operatorname{Ext}_{\mathbb{P}^{4}}^{2}(E, E)$ and itself, which is nothing but our friend $\delta_{4}$. The reason for which we chose to go through the spectral sequence anyway is to elucidate the origin of $X$ and $Y$, as we have seen, and to render transparent the Merkulov origin of $\delta_{4}$.

Summing up, we have two geometrical ways giving the same result: one coming from a more classical spectral sequence argument, another coming from a more modern perspective of $A_{\infty}$ structures.

\subsection{Obstructions}

We have so far discussed infinitesimal deformations. These are already connected to higher order terms in the superpotential, since we are expanding around a complex with non-zero maps. The power of the superpotential is indeed that deformations of a vacuum are connected to obstructions of another one.

Here we would like to push this a little further by exploring obstructions to the deformations of the complexes considered so far. It is a usual phenomenon that infinitesimal deformations do not always integrate to finite ones; from the point of view of the moduli space this is simply signaling a singularity, as the dimension of the tangent space does not equal the dimension of the manifold. In the case of a bundle, a standard reasoning says that deformations are $(0,1)$ forms $a$ that obey $\bar{\partial} a=0$, where as usual $\bar{\partial}$ includes the starting holomorphic covariant derivative on the bundle under consideration; and that first obstructions are $a \wedge a=0$ in cohomology. (Higher obstructions are called Massey products and can be again found implicitly in the $A_{\infty}$ expression for the superpotential.) Since a bundle can be also considered as a complex in derived category, this can be rephrased more generally saying that a deformation $a \in \operatorname{Ext}^{1}(C, C)$ has as first obstruction its image under the Yoneda pairing

$$
\operatorname{Ext}^{1}(C, C) \times \operatorname{Ext}^{1}(C, C) \longrightarrow \operatorname{Ext}^{2}(C, C)
$$

In terms of the representation for the $\mathrm{Ext}^{i}$ that we used in section 3 (cohomology of forms with values in complexes), this map can be expressed in the following way. If $\left(a_{1}, a_{2}, a_{3}, a_{4}\right)$ is an element of the double complex (where $a_{i} \in \Omega^{0, i-1}\left(\mathcal{M}, \oplus B_{i}^{*} \otimes B_{2-i}\right)$ ), its closure means that $d a_{1}=0, \bar{\partial} a_{1}=d a_{2}, \bar{\partial} a_{2}=d a_{3}, \bar{\partial} a_{3}=d a_{4}$, indicating collectively 
all our horizontal maps with $d$. Then one can see that the correct cocycle representing the image in $\operatorname{Ext}^{2}(C, C)$ is, apart from signs, $\left(a^{2}, a b+b a, b^{2}+a c+c a, a d+d a+b c+c b\right)$. Now, since we know the form of the cocycles associated to our modes $y$ and $x$, we can use this to derive higher orders in the superpotential. For instance, the cocycle associated to $y$ is $\left(y,\left(G \bar{\partial}^{\dagger}\right) d y,\left(G \bar{\partial}^{\dagger}\right) d\left(G \bar{\partial}^{\dagger}\right) d y,\left(G \bar{\partial}^{\dagger}\right) d\left(G \bar{\partial}^{\dagger}\right) d\left(G \bar{\partial}^{\dagger}\right) d y\right)$. Using the expression for the "square" cocycle we just gave will give higher and more complicated diagrams à la Merkulov.

\section{Gepner model and linear sigma model}

We now compute the sixth order term in the superpotential using physics methods. We follow the general approach of Diaconescu and Douglas [12], who rederived and generalized results of Recknagel and Schomerus [40] on boundary states in Gepner models using a simplified linear sigma model approach. Again, our concrete considerations are only for the quintic, but the method is general.

We start with the LG phase of the linear sigma model, which realizes the orbifold $\mathbb{C}^{5} / \mathbb{Z}_{5}$. There is also a world-sheet superpotential, but as found in [12] and as we discussed, the basic structure of the boundary states and their world-volume theory can be understood even without this. This suggests that the case of non-zero world-sheet superpotential can be treated perturbatively, as has also been found by Hori and Vafa in a slightly different context [28].

More precisely, we know from world-volume gauge invariance (at least if we do not have all five fractional branes present) that any corrections to the superpotential must contain the $Y$ fields, so all corrections vanish if the $Y$ fields are zero. In other words, the sigma model with boundary conditions defined by restricting boundary conditions from the ambient space is exactly solvable. So, we are effectively expanding in powers of the deformation away from this solvable case.

Thus we start with the free $\mathbb{C}^{5}$ theory, and the D0-brane. The massless Ramond sector of this open string theory is found by quantizing the world-sheet fermion zero modes and applying the GSO projection; this leads to states in correspondence with the odd rank $p$-forms in $\mathbb{C}^{5}$. The topologically twisted open string theory has a three-point function which is obtained by multiplying forms and taking the coefficient of the top form.

One can apply standard orbifold techniques to this theory to derive a theory with a boundary state for each irreducible representation of $\Gamma$ (the familiar "fractional branes"), a 
spectrum of massless fermions obtained by projection, and a three point function obtained by restriction.

After this preliminary discussion, we are prepared to discuss the computation of higher order terms in the superpotential from the linear sigma model. We will generally follow conventions of topological open string theory. In any case we only work with disk worldsheet, where the differences between this and the physical open string theory are minor.

The original linear sigma model action can be found in [49. The general worldsheet description of D-branes in the LG model has been discussed in [19,29] and for the linear sigma model in [20,29,22,26,27].

Since the superpotential is independent of Kähler moduli, we start by taking the limit $\zeta \rightarrow-\infty$, which takes us to the LG orbifold point. In this limit, the field $P$ gains infinite mass and we can drop it. We effectively have an LG model on $\mathbb{C}^{5} / \mathbb{Z}_{5}$, with action

$$
S=\int d^{4} \theta \sum_{i=1}^{5} \bar{\Phi}^{i} \Phi^{i}+\int d^{2} \theta W(\Phi)+\int d^{2} \bar{\theta} W^{*}(\bar{\Phi})
$$

with superpotential

$$
W=c_{i_{1} i_{2} i_{3} i_{4} i_{5}} \Phi^{i_{1}} \Phi^{i_{2}} \Phi^{i_{3}} \Phi^{i_{4}} \Phi^{i_{5}}
$$

We always use bar to denote space-time complex conjugation. The chiral superfields $\Phi^{i}$ have the standard expansion 49

$$
\Phi^{i}=\phi^{i}+\sqrt{2} \theta^{+} \psi_{+}^{i}+\sqrt{2} \theta^{-} \psi_{-}^{i}+\theta^{\alpha} \theta_{\alpha} F+\cdots
$$

We can slightly generalize the model to take independent coefficients $c$ and $\bar{c}$ of $W(\Phi)$ and $W^{*}(\bar{\Phi})$ respectively. General considerations of topological theory will tell us that correlation functions of chiral operators $O(\Phi)$ will only depend on $c$, so we will set $\bar{c}=0$.

We represent the disc as the upper half plane $z=x+i y$ with $y \geq 0$. The boundary conditions of interest to us are Dirichlet boundary conditions 19, which we take to be $\phi=\bar{\phi}=0$ and $\psi_{+}=\psi_{-}, \bar{\psi}_{+}=\bar{\psi}_{-}$. It is useful to form the combinations

$$
\begin{aligned}
\xi^{i}=\psi_{+}^{i}+\psi_{-}^{i} ; & \bar{\xi}^{i}=\bar{\psi}_{+}^{i}+\bar{\psi}_{-}^{i} \\
\tau^{i}=\psi_{+}^{i}-\psi_{-}^{i} ; & \bar{\tau}^{i}=\bar{\psi}_{+}^{i}-\bar{\psi}_{-}^{i}
\end{aligned}
$$

in terms of which the fermion boundary conditions are $\tau=\bar{\tau}=0$. Each $\bar{\xi}$ fermion will then have a zero mode (the $\xi$ zero modes do not survive the topological twisting). 
The linear sigma model has two $U(1)$ R-symmetries of which one linear combination is preserved by the boundary conditions. Under this symmetry, $\theta$ has charge $+1, d \theta$ and $D$ have charge -1 , and $\Phi$ has charge $2 / 5$. Nonzero correlation functions must include enough fermions to saturate the $\bar{\xi}$ fermion zero modes; this leads to a background $U(1)_{R}$ charge -3 .

The boundary operators we will use are(see Appendix B for more details)

$$
O_{(k ; 0)}^{i_{1} \ldots i_{k}}=\bar{\xi}^{i_{1}} \ldots \bar{\xi}^{i_{k}}
$$

and correspond to the constant $k$-forms $d \bar{\phi}^{i_{1}} \ldots d \bar{\phi}^{i_{k}}$ on $\mathbb{C}^{5}$. They have $U(1)_{R}$ charge $3 k / 5$.

The subscript 0 signifies that this is the lowest component of a superfield; one also has the one-form $d \theta O_{k}=O_{(k ; 1)}$, and a two-form $d^{2} \theta O$ from the world-sheet superpotential.

We denote $O_{(1 ; n)}=O_{X ; n}$ and $O_{(3 ; n)}=O_{Y ; n}$ as in our previous notations. Finally, we include the five-form $O_{(5 ; n)}=O_{Z ; n}$.

The quotient by $\mathbb{Z}_{5}$ is handled as in [17] and we will generally suppress these details of the computation from now on. One should also think of each boundary operator as coming with Chan-Paton factors, which can be chosen to specify any desired ordering of operators along the boundary. Equivalently, the open string couplings are free noncommuting variables. Following these considerations, each world-volume superpotential term we write is to be interpreted as a sum over terms, one for each ordering of links which leads to a closed path on the graph.

The propagators we will need are then:

$$
\begin{aligned}
\left\langle\phi^{i}\left(z_{1}\right) \partial_{y_{2}} \bar{\phi}^{j}\left(x_{2}\right)\right\rangle & =-\delta^{i j}\left(\frac{1}{z_{1}-x_{2}}-\frac{1}{\bar{z}_{1}-x_{2}}\right) \\
\left\langle\tau^{i}\left(z_{1}\right) \bar{\xi}^{j}\left(x_{2}\right)\right\rangle & =\delta^{i j}\left(\frac{1}{z_{1}-x_{2}}-\frac{1}{\bar{z}_{1}-x_{2}}\right)
\end{aligned}
$$

Only derivatives of $z$ appear, so the integrand is single-valued.

The contribution to an $n$-point correlator at $m^{\prime}$ th order in the superpotential is an integrated correlation function, with the action of $S L(2, \mathbb{R})$ divided out. The simplest example is the leading term in the world-volume superpotential, which comes from the three-point function

$$
\left\langle\left. O_{X ; 0}\right|_{0} O_{X ; 0}\left|{ }_{1} O_{Y ; 0}\right|_{\infty}\right\rangle
$$

producing

$$
\mathcal{W}=\operatorname{Tr} X^{i_{1}} X^{i_{2}} Y^{i_{3} i_{4} i_{5}} \epsilon_{i_{1} i_{2} i_{3} i_{4} i_{5}}+\ldots
$$


as found in [12,0].

The first correction will come from one insertion of the world-sheet superpotential. We will fix the $S L(2, \mathbb{R})$ symmetry by fixing the positions of two boundary operators to be $z=0$ and $z=\infty$, and taking the one-form version of the bulk operator, integrated along a contour $x \in \mathbb{R}$, $y$ fixed, which contains one point in each $S L(2, \mathbb{R})$ orbit. This leads to the insertion

$$
O_{W}=\int_{-\infty+i y}^{+\infty+i y} d x O_{(W ; 1)} \int_{-\infty+i y}^{+\infty+i y} d x c_{i_{1} i_{2} i_{3} i_{4} i_{5}} \phi^{i_{1}} \phi^{i_{2}} \phi^{i_{3}} \phi^{i_{4}} \tau^{i_{5}} .
$$

Since this is free theory, it is easy to see that this leads to the following nonzero correlation functions. The first is

$I_{1}=\left\langle O_{Y ; 0}^{j_{1} j_{2} j_{3}}(\infty) \int d x_{1} O_{X ; 1}^{k_{1}}\left(x_{1}\right) \int d x_{2} O_{X ; 1}^{k_{2}}\left(x_{2}\right) O_{Y ; 0}^{j_{1} j_{2} j_{3}}(0) \int d x_{3} O_{X ; 1}^{k_{3}}\left(x_{3}\right) \int d x_{4} O_{X ; 1}^{k_{4}}\left(x_{4}\right) O_{W}\right\rangle$

which corresponds to the term (2.11), and

$I_{2}=\left\langle O_{Z ; 0}^{j_{1} j_{2} j_{3} j_{4} j_{5}}(\infty) \int d x_{1} O_{X ; 1}^{k_{1}}\left(x_{1}\right) \int d x_{2} O_{X ; 1}^{k_{2}}\left(x_{2}\right) O_{X ; 0}^{k_{3}}(0) \int d x_{3} O_{X ; 1}^{k_{4}}\left(x_{3}\right) \int d x_{4} O_{X ; 1}^{k_{5}}\left(x_{4}\right) O_{W}\right\rangle$

whose significance we will mention later. In both cases, the limits of integration are chosen to preserve the operator ordering.

For $I_{1}$, for example, the integrand is

$$
I_{1} \propto \int_{-\infty}^{\infty} d x \int_{-\infty}^{0} d x_{2} \int_{-\infty}^{x_{2}} d x_{1} \int_{0}^{\infty} d x_{4} \int_{0}^{x_{4}} d x_{3} \prod_{i=1}^{4} f\left(x, y ; x_{i}\right) f(x, y ; 0)
$$

where

$$
f(x, y ; a) \equiv \frac{2 y}{(x-a)^{2}+y^{2}}
$$

is the Lorentzian which comes from the boundary-bulk two-point functions $-z=x+i y$ being the location of the bulk operator and $a$ the location of the boundary operator. There are five such terms in all - four from the $X$ insertions on the boundary and one from the $Y$ insertion at the origin. $S L(2, \mathbb{R})$ invariance implies that the above amplitude be independent of $y$. This is equivalent to the scale invariance of the amplitude. The Lorentzian scales as: $f(\lambda x, \lambda y ; \lambda a)=\lambda^{-1} f(x, y ; a)$. This gives a $\lambda^{-5}$ which gets cancelled from the $\lambda^{5}$ which comes from the integration measure. The scaling does not modify the limits of integration as well. 
From the expansion of the action, we have to include all allowed orderings of the $X$ 's this will lead to a combinatoric factor which is included below. By allowing the combination $x_{3}>x_{4}$ and a similar thing for $x_{1}, x_{2}$ and one can rewrite the nested integrals in unnested form (This is not quite necessary - one can also do the nested integrals to get the same result)

$$
I_{1}=\frac{1}{4} \int_{-\infty}^{\infty} d x \int_{-\infty}^{0} d x_{2} \int_{-\infty}^{0} d x_{1} \int_{0}^{\infty} d x_{4} \int_{0}^{\infty} d x_{3} \prod_{i=1}^{4} f\left(x, y ; x_{i}\right) f(x, y ; 0)
$$

where the $\frac{1}{4}$ is what remains of the $1 / 4$ ! after we take various orderings into account.

The following change of variable simplifies things: let $x=y \tan \theta$ and $x_{i}-x=y \tan \theta_{i}$. In terms of the new variables $f\left(x, y ; x_{i}\right)=y^{-1} \cos ^{2} \theta_{i}$ and the integrations over $x_{i}$ are easily done to obtain

$$
\begin{aligned}
I_{1} & =\frac{(2)^{5}}{4} \int_{-\pi / 2}^{\pi / 2} d \theta\left(\frac{\pi}{2}-\theta\right)^{2}\left(\frac{\pi}{2}+\theta\right)^{2} \\
& =\frac{4}{5} \pi^{5}
\end{aligned}
$$

The contribution of this term to the superpotential takes the form (suppressing ChanPaton labels)

$$
I_{1} c_{i_{1} i_{2} i_{3} i_{4} i_{5}} \epsilon^{j_{1} j_{2} j_{3} j_{4} i_{5}} X^{i_{1}} X^{i_{2}} Y_{j_{1} j_{2}} X^{i_{3}} X^{i_{4}} Y_{j_{3} j_{4}}
$$

which is precisely the form required to resolve the contradiction mentioned in sec. 2.6.

This expansion can of course be developed to arbitrary order. It is rather simpler than the usual interacting field theory in that there are only bulk-boundary correlators. The generalization of the term we computed to all orders takes the form

$$
\mathcal{W}=\epsilon_{i_{1} i_{2} i_{3} i_{4} i_{5}} \sum_{m \geq 0} c^{m} \operatorname{Tr}(X X Y)^{m+1}
$$

where each further term in the sum over $m$ of course involves a sum over index contractions, with coefficients determined by doing integrals of the type we discussed. Of course, the resulting coefficients need not be simple.

The $O\left(X^{5}\right)$ term should also play a role, for example in the case $(1,1,1,1,1)$. The contribution to the superpotential is of the form (suppressing Chan-Paton labels and the associated trace)

$$
I_{2} Z c_{i_{1} i_{2} i_{3} i_{4} i_{5}} X^{i_{1}} X^{i_{2}} X^{i_{3}} X^{i_{4}} X^{i_{5}} \sim I_{2} Z W(X)
$$


where $I_{2}$ is an integral (non-vanishing) similar to (4.2).

This term is interesting as we can now see a role for the field $Z$, analogous to that of the $P$-field in the world-sheet linear sigma model associated with the same CY. Namely, the equation of motion $\partial \mathcal{W} / \partial Z=0$ enforces the hypersurface condition $W=0$. It is tempting to identify this as the term in the potential which lifts degrees of freedom not lying on the hypersurface.

\section{Further examples}

We examine here the other examples we introduced in subsection 2.2, and more. For simplicity we will keep in mind the Fermat case.

We start with the RS state, which corresponds to a complex with $\left\{n_{i}\right\}=(0,0,1,2,1)$. The first thing is to write the complex analogous to (3.6), which is in this case

$$
\mathbb{C}^{1+2^{2}+1} \longrightarrow \mathbb{C}^{5} \otimes \mathbb{C}^{1 \times 2+2 \times 1} \longrightarrow \Lambda^{2} \mathbb{C}^{5}
$$

We do not a priori know which maps correspond to the RS state, but a plausible guess is that $X^{i}$ is equal to the multiplication with the vector $e_{i} \in \mathbb{C}^{2}$, and $X^{\prime} i$ as the contraction by the same vector. In this case, the cohomology is $(1,8,3)$. This tells us that on $\mathbb{P}^{4}$ the complex would have moduli space of dimension 8; we now have to check how many of the candidate extra 3 moduli add to these when restricting to $\mathcal{M}$.

First of all, the equation $X \cdot y=0=y \cdot X^{\prime}$ becomes now $\epsilon_{i j k l 1} y^{i j k}=0=\epsilon_{i j k l 2} y^{i j k}$; this leaves only $y_{12 i}, i \neq 1,2$ : these are the three candidates. The real check is now again $\delta_{4}$. In this case the situation is more interesting: in components one can see that the quintic term is now $\left(X^{2} X^{\prime 2} y\right)^{i j}=y^{1 i j}+y^{2 i j}$. So the meaningful equations are obtained setting one of the $i, j$ equal to 1 or 2 :

$$
0=x_{1}^{i}+y^{1 i 2}=x_{2}^{i}+y^{2 i 1}=x_{1}^{i i}+y^{i 12}=x_{2}^{\prime i}+y^{i 21}
$$

(remember that $x$ and $x^{\prime}$ are each element of $\mathbb{C}^{5} \otimes \mathbb{C}^{2}$, and so they have an extra lower index taking values 1 or 2 ). None of these equations set a $y$ field to zero: in each case, there is the possibility of switching on a companion $x$ field in such a way that the equation is satisfied without having to put the $y$ to zero. We can again rephrase this in a dual picture: The result $y^{1 i j}+y^{2 i j}=\left[\delta_{4}(y)\right]_{i j}$ is not "gauge fixed", in the sense that it is not orthogonal to $X$ and $X^{\prime}$ : those which are are of the form $y^{12 i}$, as we already know. So when we try to 
project $y^{1 i j}+y^{2 i j}$ to the space of the $y^{12 i}$, as we did in (3.11), we get zero: this is exactly the dual of the fact that these equations can be "gauged away" by introducing the extra $x$ and $x^{\prime}$. Either way, this means that the 3 fields are in the kernel of $\delta_{4}$. This predicts that this complex should have in total $8+3=11$ moduli on $\mathcal{M}$, which is consistent with the Recknagel-Schomerus computation.

We now come to the other class of examples we introduced in subsection 2.2, that is $\mathcal{O}(i)_{\left.\right|_{\mathbb{P}^{j}}}$. This class of examples is easy to compute using the Beilinson procedure because the cohomology groups of the $\mathcal{O}(i)$ are very well known. In general, it is better to reduce the result of this procedure to one line, in order to have a simple complex of $B_{i}$, which leads to a quiver representation. In the class of examples at hand, this happens if $i+j \geq 4$. It turns out interesting however to also break this rule; the resulting example is interesting and we will describe it in a little more detail, whereas for the other cases we only describe the results.

The first case we handle is $\mathcal{O}(2)_{\left.\right|_{\mathbb{P} 3}}$. In this case $\left\{n_{i}\right\}=(0,0,1,4,10)$ and so the starting complex is $\left(1+4^{2}+10^{2}\right) \longrightarrow 5 \times(1 \times 4+4 \times 10) \longrightarrow 10 \times(1 \times 10)$; as opposed to the RS case above, we can write down the maps explicitly here with no guesswork, and the cohomologies result to be $(1,4,0)$. This means that there are $4 X$ and no $Y$; so it means that to the 4 moduli that the sheaf had on $\mathbb{P}^{4}$ (which are right, because a hyperplane $\mathbb{P}^{3} \subset \mathbb{P}^{4}$ has 4 moduli and the $\mathcal{O}(2)$ on it is rigid) nothing adds when restricting to the Calabi-Yau. So in this case we did not really need our methods.

Something more interesting arises if one considers instead $\mathcal{O}(2)_{\left.\right|_{\mathbb{P}^{2}}}$, whose numbers are $\left\{n_{i}\right\}=(0,0,1,3,6)$. The complex is $\left(1+3^{2}+6^{2}\right) \longrightarrow 5 \times(1 \times 3+3 \times 6) \longrightarrow 10 \times(1 \times 6)$ and its cohomology is $(1,6,6)$. This case is more interesting as in addition to the 6 moduli on $\mathbb{P}^{4}$ (again these are right, as this is the dimension of a Grassmannian $G(3,5)$ ), there are 6 more candidate moduli upon restricting to $\mathcal{M}$. As in the RS case we can write down the explicit equations and we discover in a similar way that all the moduli are not lifted. So we have $6+6=12$ moduli for this sheaf on $\mathcal{M}$. How can we check that this is right geometrically? Viewing this sheaf as a bundle on its support, as usual we have to count deformations of the support inside the Calabi-Yau $\mathcal{M}$ and deformations of the line bundle on the curve $\mathbb{P}^{2} \cap \mathcal{M}$, which is a Riemann surface $C$. First type of deformations are at least six, as moduli that were there on $\mathbb{P}^{4}$ cannot be lifted. On the other hand, $C$ can be viewed as a curve of degree 5 inside $\mathbb{P}^{3}$, and so has genus 6 . Deformations of line bundles on a Riemann surface are exactly as many as the degree, and so we have 6 other 
deformations from here. We know that 12 is the maximum allowed, and so we get that it is the right number.

It would now be natural to consider something which in $\mathbb{P}^{4}$ has support $\mathbb{P}^{1}$, in such a way that we get 5 D0 branes on the quintic. According to the rule we expounded above, to have a single quiver representation we should now consider at least $\mathcal{O}(3)_{\left.\right|_{\mathbb{P} 1}}$. This would give a longer complex, examples of which we will consider shortly. We can however remain in the more familiar domain of complexes of length 3 by considering, instead of a single quiver representation, a more generic complex of quiver representations: explicitly $q_{1} \equiv(0,0,1,2,3) \longrightarrow q_{2} \equiv(1,0,0,0,0)$. This is a little off the track in that an analysis with a double complex similar to what we did in section 3 for sheaves shows that this object is not simple (Hom $>1)$, and deformations may also come from a $\operatorname{Ext}^{2}\left(q_{2}, q_{1}\right)$ that we should compute. It is interesting however to check what are the deformations of the $\operatorname{Ext}^{1}\left(q_{1}, q_{1}\right)$. There are in this case $6 X$ and $9 Y$; none of these $Y$ is lifted by our superpotential, so that the total moduli are $6+9=15$. This is perfectly consistent with fact that the total object represents 5 D0 on the Calabi-Yau.

\subsection{Longer complexes}

So far we have restricted ourselves to examples of complexes for which only 3 consecutive nodes were present. The reason this is simpler can be seen in terms of the double complex. For longer complexes, the complex (3.4) would again be present only for $p=0$ and 3 , but it will be longer in both directions, because there will now be bundles $B_{5}^{*} \otimes B_{1}$ and $B_{1}^{*} \otimes B_{5}$. In the general case $\left(n_{1}, \ldots, n_{5}\right)$, the zeroth line (3.6) will read

$$
\mathbb{C}^{\Sigma n_{i}^{2}} \longrightarrow V \otimes \mathbb{C}^{\Sigma n_{i} n_{i+1}} \longrightarrow \Lambda^{2} V \otimes \mathbb{C}^{\Sigma n_{i} n_{i+2}} \longrightarrow \ldots \longrightarrow \Lambda^{4} V \otimes \mathbb{C}^{n_{1} n_{5}}
$$

We will start by looking at the example $(0,1,1,1,1)$, which should be the anti brane to $B_{1}$ and thus rigid on $\mathcal{M}$. In this case $E^{2}$ is (compare (3.7))

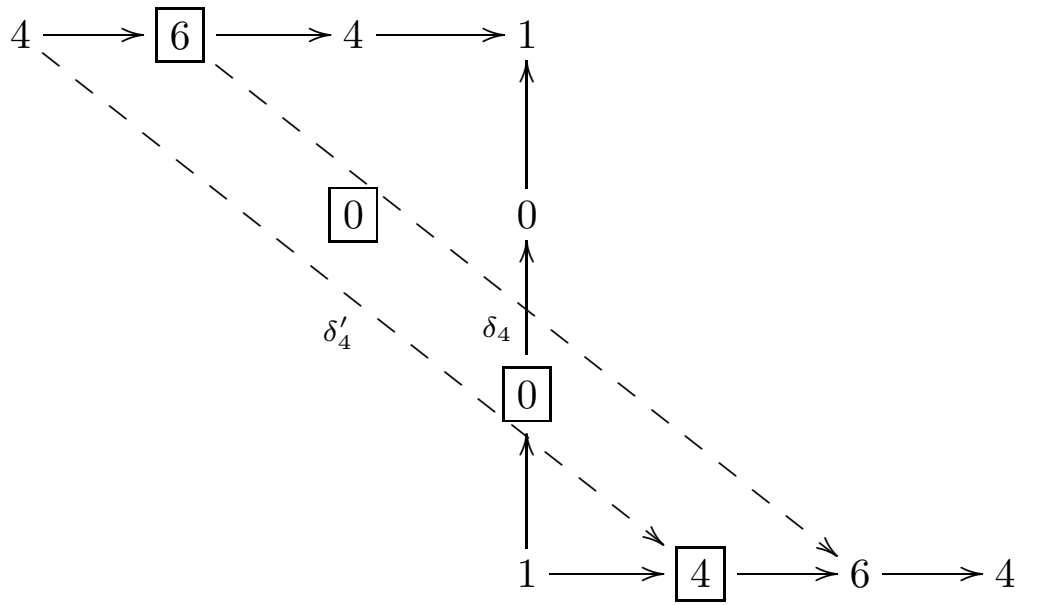


What happens here is that both $\delta_{4}, \delta_{4}^{\prime}$ are isomorphisms, and lift both $y$ and $x$ deformations. So we already see from this example that the comparison to the procedure for computing deformations and Hom of quiver representations we performed in section 3 is less evident. This is not a surprise if we get back at our exact sequence (2.8). Remember that, as we outlined at the end of section [3, we can recover this exact sequence from the spectral sequence via an argument à la Gysin for our spherical bundles. Then we see that a larger spectral sequence gives rise to non-zero $\operatorname{Ext}_{\mathbb{P}^{4}}^{1}\left(E, E^{\prime}\right)$ that complicate the story exactly as it happens in lower dimensions, see remarks after (2.8).

We end with two natural continuations of the examples discussed so far. One is $(1,1,1,1,1)$, for which zeroth line is this time $1 \longrightarrow 4 \longrightarrow 6 \longrightarrow 4 \longrightarrow 1$ and there are three maps $\delta_{4}$ this time. All of them are isomorphisms, and this time even Hom gets cancelled! This is not surprising if we remember that this state is the D0 on $\mathbb{P}^{4}$, and so it restricts to nothing on $\mathcal{M}$.

Our last example will be the more straightforward realization of the state with 5 D0 branes on $\mathcal{M}$ that we anticipated above, obtained as $\mathcal{O}(3)_{\left.\right|_{\mathbb{P} 1}}$. This time we get

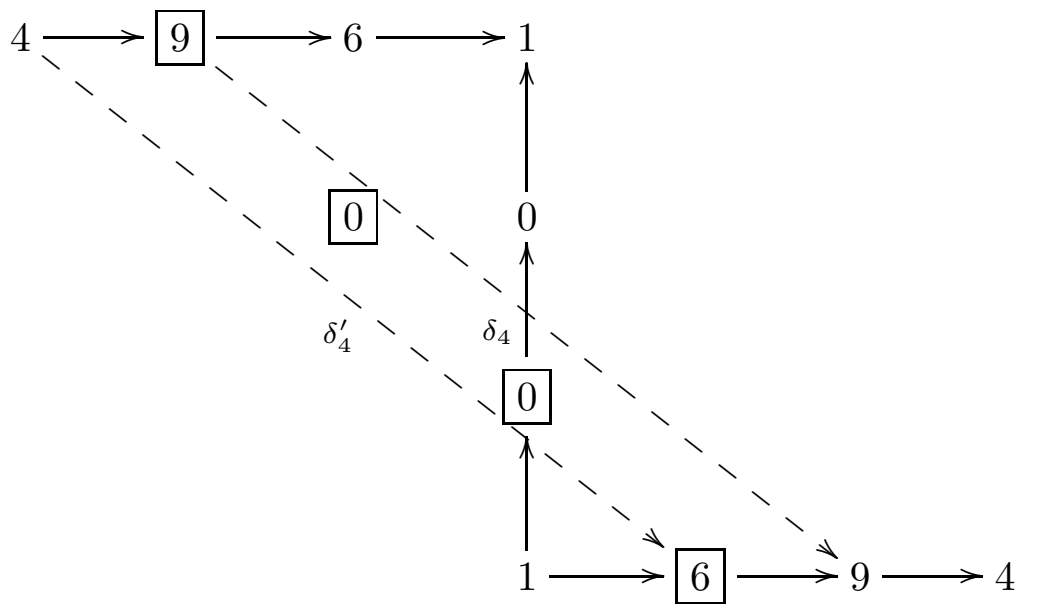

We have this time that both $\delta_{4}, \delta_{4}^{\prime}$ are zero; this implies that deformations are $6+9=15$ as in the previous 5 D0 realization; moreover, here we have that the Hom are $1+4=5$, so the 5 D0 are not bound, but each contributes its own unbroken gauge groups.

Let us note that in all the examples we have seen, either all or none of the $Y$ are lifted. The reason for this is that the original double sequence has a symmetry $(p, q) \longrightarrow(-p, 3-q)$, which one can use to construct the same arrow in reverse. This means that as long as this symmetry is not broken the map (once projected as in (3.11)) can be written as $\left[\delta_{4}(y)\right]_{i j}=\lambda y_{i j}$, so is either the zero map or an isomorphism. 


\subsection{Conclusions}

We saw in these examples that the framework as we described it appears to be a satisfactory description of bound states involving three types of fractional brane. It is not obvious at this point whether higher order corrections of the form (4.5) are required to get exact results. Besides a direct approach, one might gain information on this point by comparing our results with those of [10], who get information on the spectrum of BPS branes from a very different starting point (supergravity and the attractor mechanism).

We discussed bound states of more fractional branes as well. Mathematically, these examples work rather similarly to the previous ones, with the non-trivial aspects of the restriction captured in the maps $\delta_{4}$, information which we believe can again be summarized in the superpotential. What changes is only that the pairing now involves additional Hom's between the complexes, which appear on restriction.

In fact, a naive approach to modeling these bound states as supersymmetric quiver gauge theory involving four or five nodes, does not work. The basic problem is that one does not see the extra Hom's in this description. These are additional brane-antibrane tachyons which must appear because of identifications such as $B_{1}+B_{2}+B_{3}+B_{4}=\bar{B}_{5}$. Similar problems with the naive application of supersymmetric gauge theory to problems with too many different fractional branes were already observed in [12]; in particular one cannot reproduce the correct bosonic masses using Fayet-Iliopoulos terms.

One way to deal with this would be to forget about supersymmetric gauge theory (i.e. abelian categories) and instead always work with the derived category. Another possibility is to apply Seiberg dualities to some of the fractional branes [4], to try to get valid supersymmetric field theories in these cases. Clearly systematizing this is an important question for future work.

\section{Conclusions and further directions}

In this work, we have taken a further step in the development of a concrete framework which can describe all BPS D-branes in Calabi-Yau compactification of string theory, by showing how to handle a compact Calabi-Yau.

The framework can be motivated and justified both from physics and mathematics starting points, and we have shown at length how the two points of view are related. Not being mathematicians, we will summarize what we have done from this point of view very succinctly. In principle, the obstruction theory of the restriction of the derived 
category of coherent sheaves from $\mathbb{P}^{4}$ to a quintic hypersurface, could be obtained from computations in holomorphic Chern-Simons theory. In practice such computations are difficult. In section 4 of this work, we define a topological field theory which encodes the same information, but in which computations are straightforward, and exhibit the first non-trivial correction.

From a physics point of view, we start with certain fractional branes at the LandauGinzburg orbifold point of the linear sigma model. These are a particularly simple set of constituents from which a large subset of all branes can be constructed as bound states, by finding vacua of their supersymmetric world-volume theories (and generalizations of this prescription). In previous work, it was shown that by taking a cubic world-volume superpotential, one precisely describes the branes which correspond to sheaves on the resolved orbifold, corresponding to the linear sigma model with zero world-sheet superpotential. Adding the world-sheet superpotential constructs the CY as a hypersurface in this resolved orbifold, and our primary question was to find out whether the same type of description is valid and what precise modifications the world-sheet superpotential induces.

We studied the restriction to the hypersurface in some detail using homological algebra and spectral sequence techniques, justifying the claim that in three and more complex dimensions branes on CY are very similar to those on the ambient space, so that the world-sheet superpotential can be treated perturbatively. From a physics point of view this might be considered surprising, as the world-sheet superpotential induces a non-trivial RG flow from (in our case) $\hat{c}=5$ to $\hat{c}=3$. Nevertheless it clearly works in the problem at hand, and this is one of the important lessons of our work.

Perhaps the most conservative explanation of why perturbation theory in the worldsheet superpotential works here, is that our D-branes are all localized to the orbifold singularity, and after the resolution become branes wrapping cycles in $\mathbb{P}^{4}$; thus the sector of the theory we consider has fewer light degrees of freedom than a generic $\hat{c}=5$ string. Of course it might be that it works in greater generality.

The restriction to the CY does change the detailed properties of the branes. On physical grounds, most of these modifications can be expressed by corrections to the worldvolume superpotential. We computed the leading such correction, which contains the leading dependence on the complex structure of the CY, and showed both on general grounds and in examples that this correctly describes the modifications to the category of coherent sheaves produced by restriction. Thus, we see that branes on compact CY's can be described by these methods. 
Although we only discussed the case of the quintic, as we mentioned in the introduction these methods generalize to large classes of CY's which can be realized in the linear sigma model framework, and the new linear sigma computations we have done appear no harder in the general case.

Can we hope that these techniques could lead to a complete description of branes on CY? There are numerous issues to be understood.

The first is that strictly speaking one only gets a subset of the K theory, those classes which can be obtained by restriction. This is probably a minor problem as one can take limits in which these objects become reducible, splitting into objects with the other classes. For example, although one cannot obtain the D0 on the quintic, one can intersect a curve with the defining surface to obtain five D0's; the formalism then produces the additional moduli required to move these independently.

The second problem is that one is still working by expansions around more solvable points, and some phenomena are hard to see this way. For example, one expects moduli spaces of branes on compact CY to have some type of compactness. In this description, the compactness of moduli associated to the ambient space (the $X^{\prime}$ 's) is manifest; however one also expects compactness in the $Y$ directions, which correspond to field identifications which will not be obvious. At this point, this is probably the most serious problem we foresee. Such field identifications will be signaled however by new light states (e.g. consider the theory of two D0's on a torus) which might well have some signature in the superpotential, so it is too early to say whether this can be better understood.

In section 5 we mentioned the problem that quiver theories including too many different fractional branes cannot be treated in a naive way as supersymmetric gauge theories (a signal of this is that the sum of bosonic squared masses around a closed loop is non-zero, which cannot be reproduced by Fayet-Iliopoulos terms). We believe that this problem goes away upon more correctly treating brane-antibrane combinations, either in the derived category or by performing appropriate Seiberg dualities. This point also deserves to be better understood, but is probably not an obstacle.

Finally, of course a complete solution of this problem with these ideas would seem to rest on being able to find the exact superpotential. Now, there are many highly nontrivial exact superpotentials known for $\mathcal{N}=1$ supersymmetric theories, so in itself we consider this a rather encouraging reformulation of the problem.

As we discussed, this superpotential can also be considered as a rewriting of the holomorphic Chern-Simons action in a more explicit form, depending on a finite number 
of variables. We have essentially proposed an alternate topological open string theory which is more tractable than the original one. This theory is rather similar to that developed by Cattaneo and Felder [9] to describe deformation quantization, and particularly to their generalizations with higher rank forms. Many mathematical connections between these problems are known. 43

There is also a close analogy between this superpotential and the periods of the holomorphic three-form which were the original subject of mirror symmetry. Indeed, the superpotential for a wrapped two-brane is a semiperiod [13,50] which in interesting examples [1] satisfies generalized Picard-Fuchs equations for which the periods are also solutions [23, 34]. Various extensions of this relation are known, suggesting that the coefficients of the series expansion (4.5) should be closely related to those in the comparable series expansions of the periods [8], or that the superpotential itself will satisfy a linear differential equation.

For all of these reasons, we believe that the further study of this superpotential will be a fruitful direction in the near future. Here we explained how this would lead to a concrete description of the derived category of sheaves on CY, which would have numerous applications in physics and mathematics.

We acknowledge valuable discussions with D.-E. Diaconescu, R. Dijkgraaf, K. Paranjape, and especially with P. Seidel. We also thank B. Szendroi for critical remarks, which

are addressed in version 2. T. J. and A. T. would like to thank the NHETC of Rutgers University for its kind hospitality during first phases of this project.

This work was supported by DOE grant DE-FG02-96ER40959.

\section{Appendix A. The standard complex for quivers}

We describe this here both to show that our "intuitive" way of computing deformations of quivers does not hide mathematical subtleties, but also to show how to compute higher Ext. The degree of complication we will meet should make one appreciate the power of generalized McKay correspondence, that allows one to compute such groups by means of more standard geometrical procedures. We will start from abstract definitions and try to get quickly to examples, to show how it works.

Given our quiver $Q$, let us denote its nodes by $d_{0}, \ldots, d_{k}$; as for arrows between $d_{i}$ and $d_{j}$, we will consider them as formal generators of abelian groups; this group we dub $Q\left(d_{i}, d_{j}\right)$. (This realizes what is called a $\mathbb{Z}$-linear category.) The standard complex is then

$$
\begin{gathered}
\ldots \oplus_{i, j} Q\left(d_{i}, d^{\prime}\right) \otimes Q\left(d_{j}, d_{i}\right) \otimes Q\left(d, d_{j}\right) \longrightarrow \oplus_{i} Q\left(d_{i}, d^{\prime}\right) \otimes Q\left(d, d_{i}\right) \longrightarrow Q\left(d, d^{\prime}\right) \\
a \otimes b \otimes c \mapsto a b \otimes c-a \otimes b c
\end{gathered}
$$


where all tensor products are over $\mathbb{Z}$, and a map has been shown for illustration. The importance of this complex is that, given any quiver representation $M$, tensoring with this complex gives a projective resolution of it:

$$
\oplus_{i, j} Q\left(d_{j}, ?\right) \otimes Q\left(d_{i}, d_{j}\right) \otimes M\left(d_{i}\right) \longrightarrow \oplus_{i} Q\left(d_{i}, ?\right) \otimes M\left(d_{i}\right) \longrightarrow M
$$

here, for instance, central term is a representation whose vector space corresponding to node $d$ is $\oplus_{i} Q\left(d_{i}, d\right) \otimes M\left(d_{i}\right)$. We will see in a moment how this works. Before of that, let us also recall that the $\operatorname{Ext}^{i}$ of two quiver representations $M$ and $N$ are then obtained taking $\operatorname{Hom}(\cdot, N)$ of (A.1), and considering the cohomology of the resulting complex. We obtain:

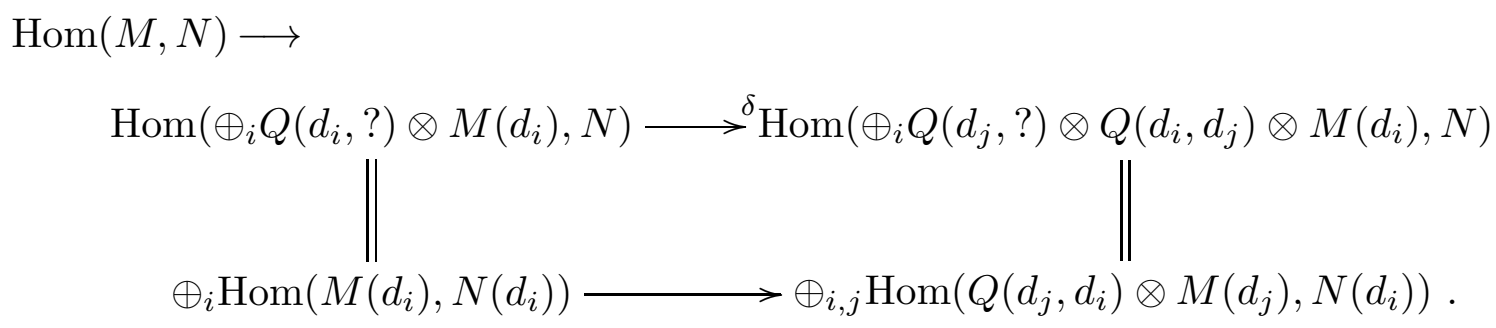

and so on. The two vertical equalities are because of something called Yoneda lemma. We will illustrate it in a baby example, which will takes us on our way to more grown-up ones shortly. Consider a quiver $d_{1} \stackrel{\alpha}{\longrightarrow} d_{2}$, and the representation of it $Q\left(d_{1}, ?\right)$, where $d_{1}$ is its first node. This representation is simply the one whose vector space at each node is generated by the arrows starting from $d_{1}$; in this case, at $d_{1}$ itself we only have $e_{d_{1}}$, the identity on $d_{1}$, whereas at $d_{2}$ we have $\alpha$. This representation can be thus written more concretely as $1 \stackrel{1}{\longrightarrow} 1$. If we consider now quiver morphisms of this into any other representation $N$,

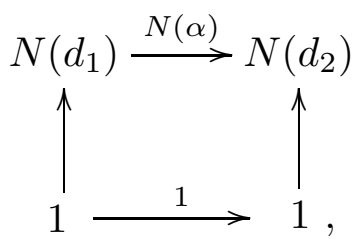

then we have as our only choice the image of the lower left 1 inside $N\left(d_{1}\right)$, call it $n_{1}$. Indeed the image of the other 1 then follows to be simply $n_{2}=N(\alpha)\left(n_{1}\right)$ since the diagram should commute. So we have got an explicit isomorphism $\operatorname{Hom}\left(Q\left(d_{1}, ?\right), N\right) \stackrel{\sim}{\longrightarrow} N\left(d_{1}\right)$; more generally the same mechanism yields

$$
\operatorname{Hom}(Q(d, ?), N) \stackrel{\sim}{\longrightarrow} N(d)
$$


for $Q$ whatever quiver and $d$ whatever node.

What we want to do now is to illustrate the maps in (A.2), so that it become explicit to compute its first cohomology. We consider now the quiver $d_{1} \stackrel{\alpha}{\longrightarrow} d_{2} \stackrel{\beta}{\longrightarrow} d_{3}$. We start by studying the map $\delta$ in (A.2). Its source space is in this case given by homomorphisms of representations:

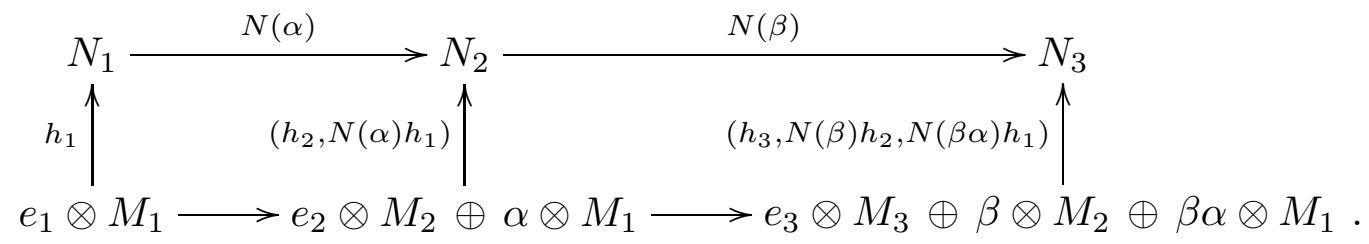

Here the $h_{i}$ can be simply seen as elements of $\operatorname{Hom}\left(M\left(d_{i}\right), N\left(d_{i}\right)\right)$, as another manifestation of Yoneda lemma, compare again with (A.2). Our aim is then to find the image of this. Elements of the arrival space of $\delta$ are now multiples $\left(a_{1}, a_{2}, a_{3}, b_{1}, b_{2}, c_{3}\right)$, which can be seen as candidate deformations (in the case $M=N$, of course) of the given representations for $e_{1}, e_{2}, e_{3}, \alpha, \beta, \beta \alpha$. The map we are going to find is then the identification of different deformations; we will consider afterwards their consistency as candidate deformations (closure under the following map). Also for these would-be deformations $a_{i}, b_{i}, c$ a representation of the type we gave in (A.3) for $\left(h_{1}, h_{2}, h_{3}\right)$ can be given. On the lower right corner we have now

$$
e_{3} \otimes e_{3} \otimes M_{3} \oplus e_{3} \otimes \beta \otimes M_{3} \oplus \beta \otimes e_{3} \otimes M_{3} \oplus e_{3} \otimes \beta \alpha \otimes M_{3} \oplus \beta \otimes \alpha \otimes M_{3} \oplus \beta \alpha \otimes e_{3} \otimes M_{3}
$$

which goes into $N_{3}$ through a map $\left(a_{3}, b_{2}, N(\beta) a_{2}, c, N(\beta) b_{1}, N(\beta \alpha) a_{1}\right)$ (compare again with (A.3)). An element $e_{3} \otimes e_{3} \otimes m_{3}$ in the first summand of (A.4) is now sent by the map in the standard complex to $\left(e_{3} e_{3} \otimes m_{3}-e_{3} M\left(e_{3}\right) \otimes m_{3}\right)=0$. An element $e_{3} \otimes \beta \otimes m_{3}^{\prime}$ in second summand is already more interesting, being sent into $\beta \otimes m_{3}^{\prime} \oplus\left(-e_{3} M(\beta) m_{3}^{\prime}\right)$. To this we can apply third vertical map in (A.3) getting $N(\beta) h_{2}-h_{3} M(\beta)$ ! Here we recognize nothing but the infinitesimal action of change of basis on nodes $d_{2}$ and $d_{3}$ on the representation of the arrow $\beta$ (again, in view of the case $M=N$ which is our main application here). Going on in this way we find as image of $h_{i}$ the multiple defined by

$a_{i}=0, \quad b_{1}=N(\alpha) h_{1}-h_{2} M(\alpha) \quad b_{2}=N(\beta) h_{2}-h_{3} M(\beta), \quad c=N(\beta \alpha) h_{1}-h_{3} M(\beta \alpha)$.

This is good, but the really interesting thing is to check the way deformations are considered as valid ones, more than the identifications among them. A cursory look at 
what we just described will convince anyone that writing everything explicitly would be not particularly readable. But we can describe salient pieces. We start now from a multiple $a_{i}, b_{i}, c$, and look for their images $A_{i}, B_{i}, C_{i}, D$ which have then to be put to zero to ensure closure. The analogue of (A.4)has now 10 summands, each with 4 "factors". Let us consider one of them, the tensor product $e_{3} \otimes \beta \otimes \alpha \otimes M_{1}$. An element of it undergoes now

$$
\begin{aligned}
e_{3} \otimes \beta \otimes \alpha \otimes m_{1} & \mapsto \beta \otimes \alpha \otimes m_{1} \oplus\left(-e_{3} \otimes \beta \alpha \otimes m_{1}\right) \oplus e_{3} \otimes \beta \otimes M(\alpha) m_{1} \\
& \mapsto\left[N(\beta) b_{1}-c+b_{2} M(\alpha)\right] m_{1} .
\end{aligned}
$$

A more complete analysis reveals that closure amounts to put to zero $a_{i}$ and the quantity inside brackets to zero. This relation reexpresses the deformation $c$ of $\beta \alpha$ in terms of deformations $b_{i}$ of $\beta$ and $\alpha$, leaving them unconstrained otherwise. Thus we have that deformations are given by the two $b_{i}$, divided by the relations we already found. This is exactly what we would have done intuitively.

Actually, for quiver without relations we could have resorted to the more handy Hom complex provided by Kac [30] (for a review see [15]). So the method comes into its own for the case with relations. Let us then modify the quiver we just saw introducing a relation $\beta \alpha=0$. We can repeat the computation we just did with a few obvious changes: in particular the term with a - sign in (A.5) drops, leading instead to a relation $N(\beta) b_{1}+$ $b_{2} M(\alpha)=0$. For $M=N$, this is just what we would get from the "naive" procedure used in the text: considering

$$
\left(M(\beta)+b_{2}\right)\left(M(\alpha)+b_{1}\right)=0
$$

to first order (zero order is obviously the representation of the relation).

For other finite quivers (having a finite number of paths) these computations are not really more difficult. In particular, one can check that for the Beilinson quiver the standard complex can be simplified to yield the simpler procedure considered in 15].

The fact that our quivers are not finite, instead, would make repeating this procedure explicitly in our case would be instead awfully complicated. Even considering only the portion we are using of the McKay quiver (only three consecutive nodes are present), the relations derived from the superpotential do not make finite the possible paths: indeed there are for instance loops like $y_{45} x_{1}^{\prime} x_{1}$, and so on. So already writing the simplest thing, (A.3), would consist in this case of infinite pieces in each of the three nodes (indeed because of loops like the one just mentioned). However, the principles we abstracted from the finite examples we considered in some detail here are that: 
1 - a deformation of a composite path can be reexpressed in terms of deformations of the constituents (compare the square bracket in (A.5))

2 - when there is a relation, its effect can be reckoned as coming from the simple intuitive procedure (A.6).

In particular first principle allows us to consider only deformations of generators of the possible paths, if we take care of the relations according to second principle.

\section{Appendix B. The topological LG model}

Consider a LG model with chiral superfields $\Phi^{i}$ and a quasi-homogeneous superpotential $W(\Phi)$. As shown by Witten, this model can be obtained as a phase of the gauged linear sigma model. For LG models on worldsheets with boundary preserving B-type supersymmetry, it has been shown[19] 29] that the only boundary conditions are those compatible with the condition $W=0$. In simple models involving a single chiral field, the only possible condition is the Dirichlet one. This carries over to the case of several chiral superfields when one imposes boundary conditions separately on each of the chiral superfields. For LG models which flow in the infrared to Gepner models associated with Calabi-Yau compactifications, this implies that all the Recknagel and Schomerus boundary states must necessarily arise from Dirichlet conditions being imposed on each of the chiral superfields. Further, when the superpotential is degenerate at $\phi^{i}=0$, the condition $W=0$ implies that the RS states arise from the boundary condition $\phi^{i}=0$. The boundary conditions that we will consider in the LG model is given by

$$
\phi^{i}=0 \quad, \quad \tau^{i} \equiv\left(\psi_{+}^{i}-\psi_{-}^{i}\right)=0,
$$

where the fermionic boundary condition is obtained by closure under the unbroken supersymmetry. Let us parametrise the unbroken supersymmetry $\epsilon=\sqrt{2} \epsilon_{+}=\sqrt{2} \epsilon_{-}$under which the other components of the chiral superfield transform as

$$
\begin{gathered}
\delta \xi^{i}=2 i \bar{\epsilon} \partial_{y} \phi^{i} \\
\delta\left(\partial_{y} \phi^{i}\right)=-i \epsilon \partial_{x} \xi^{i},
\end{gathered}
$$

where $\xi^{i} \equiv\left(\psi_{+}^{i}+\psi_{-}^{i}\right)$. It follows that $\left(\xi^{i}, \sqrt{2} i \partial_{x} \phi^{i}\right)$ form an antichiral fermi superfield (in boundary superspace with $\theta \equiv-\sqrt{2} \theta^{+}=\sqrt{2} \eta \theta^{-}$)

$$
\Xi^{i}=\xi^{i}-2 \bar{\theta} i \partial_{y} \phi^{i}-\theta \bar{\theta} \partial_{x} \xi^{i}
$$


Note that we have made use of the fact that $W$ is degenerate at $\phi^{i}=0$ and thus one has $F_{i}=0$ on the boundary. Further, we also need to use the bulk equations of motion for the fermions.

Let us now specialise to the case of the quintic where we have five chiral superfields $\Phi^{1}, \cdots, \Phi^{5}$ and a degree five superpotential. The boundary interactions take the form

$$
\int d \theta\left(X^{i} \bar{\Xi}^{i}+Y^{i j k]} \bar{\Xi}^{i} \bar{\Xi}^{j} \bar{\Xi}^{k}+Z \bar{\Xi}^{1} \bar{\Xi}^{2} \bar{\Xi}^{3} \bar{\Xi}^{4} \bar{\Xi}^{5}\right)+h . c .
$$

$X, Y$ and $Z$ are Chan-Paton matrices. The assignment of appropriate Chan-Paton factors is not immediately obvious from the topological LG considerations above. This requires a careful consideration of allowed fractional brane states and the states appearing in openstrings connecting them as in [12].

\section{B.1. The topological model}

Consider the topological twist where $\bar{\epsilon}$ becomes a scalar. It follows that in this topological theory, terms which appear in the action as the following superspace integrals $\int d^{4} \theta$ or $\int d^{2} \bar{\theta}$ or $\int d \bar{\theta}$ are BRST exact. Hence, the topological theory depends solely on the holomorphic superpotential $W(\phi)$ in the bulk and $\mathcal{X}^{i}, \mathcal{Y}^{i j k}$ and $\mathcal{Z}$ couplings in the boundary.

In the bulk, any holomorphic function such as $W(\phi)$ is a topological observable which is a zero-form operator $O_{(W ; 0)}$ in the bulk. Its one-form and two-form versions are obtained by the usual descent procedure. They are given by

$$
\begin{aligned}
& O_{(W ; 1)}=\frac{\partial W}{\partial \phi^{i}} \tau^{i} \text { and } \frac{\partial W}{\partial \phi^{i}} \xi^{i} \\
& O_{(W ; 2)}=\frac{\partial^{2} W}{\partial \phi^{i} \phi^{j}} \tau^{i} \xi^{j}+2\left|\frac{\partial W}{\partial \phi^{i}}\right|^{2}
\end{aligned}
$$

where $\tau^{i} \leftrightarrow \partial_{x} \phi^{i} d x$ and $\xi^{i} \leftrightarrow \partial_{y} \phi^{i} d y$.

On the boundary, analogously, one has zero-form and one-form observables. They are

$$
\begin{aligned}
& O_{(k ; 0)}^{\left[i_{1} \ldots i_{k}\right]}=\bar{\xi}^{i_{1}} \ldots \bar{\xi}^{i_{k}} \\
& O_{(k ; 1)}^{\left[i_{1} \ldots i_{k}\right]}=2 i \partial_{y} \bar{\phi}^{\left[i_{1}\right.} \bar{\xi}^{i_{2}} \ldots \bar{\xi}^{\left.i_{k}\right]}
\end{aligned}
$$

The index $k$ indicates that they correspond to $k$-forms $d \bar{\phi}^{i_{1}} \ldots d \bar{\phi}^{i_{k}}$ on $\mathbb{C}^{5}$. Only odd-forms survive the GSO projection and these are boundary condition changing operators (except when $k=5)$. 
The ghost number in the topological theory is the same as the unbroken $U(1)_{R}$ charge. The ghost numbers assignments are

$$
\begin{array}{ccccc}
\text { Field } & \phi & \tau & \xi & F \\
\text { Ghost No. } & \left(0+\frac{2}{5}\right) & \left(-1+\frac{2}{5}\right) & \left(-1+\frac{2}{5}\right) & \left(-2+\frac{2}{5}\right)
\end{array}
$$

where the shift of $2 / 5$ is the modification due to the addition of a homogeneous superpotential of degree five[49]. 


\section{References}

[1] M. Aganagic and C. Vafa, "Mirror Symmetry, D-Branes and Counting Holomorphic Discs," arXiv:hep-th/0012041.

[2] P. S. Aspinwall and M. R. Douglas, "D-brane stability and monodromy," arXiv:hepth/0110071].

[3] A. A. Beilinson, "Coherent sheaves on $\mathbb{P}^{n}$ and problems of linear algebra", Funct. Anal. Appl. 12 (1978) 214-216.

[4] D. Berenstein and M. R. Douglas, work in progress.

[5] R. Bott and L. W. Tu, Differential Forms in Algebraic Topology, Springer, 1982.

[6] I. Brunner, M. R. Douglas, A. Lawrence, and C. Römelsberger, "D-branes on the quintic,", JHEP 0008, 015 (2000) arXiv:hep-th/9906200.

[7] I. Brunner and V. Schomerus, "On superpotentials for D-branes in Gepner models," JHEP 0010 (2000) 016 arXiv:hep-th/0008194.

[8] P. Candelas, X. C. de la Ossa, P. S. Green, and L. Parkes, "A pair of Calabi-Yau manifolds as an exactly soluble superconformal theory", Nucl. Phys. B359 (1991) 21.

[9] A. S. Cattaneo and G. Felder, "A path integral approach to the Kontsevich quantization formula," Commun.Math.Phys. 212 (2000) 591-611; math.QA/9902090.

[10] F. Denef, B. R. Greene and M. Raugas, "Split attractor flows and the spectrum of BPS D-branes on the Quintic," JHEP 0105 (2001) 012; hep-th/0101135.

[11] D.- E. Diaconescu, "Enhanced D-branes categories from string field theory," , JHEP 06 (2001) 016 arXiv:hep-th/0104200.

[12] D.-E. Diaconescu and M. R. Douglas, "D-branes on Stringy Calabi-Yau Manifolds," arXiv:hep-th/0006224.

[13] S. K. Donaldson and R. P. Thomas, "Gauge theory in higher dimensions," in: The Geometric Universe; Science, Geometry and the work of Roger Penrose, eds. S. A. Huggest et al, Oxford Univ. Press 1998.

[14] M. R. Douglas, "D-branes, categories and N=1 supersymmetry," arXiv:hep-th/0011017.

[15] M. R. Douglas, B. Fiol, and C. Römelsberger, "The spectrum of BPS branes on a noncompact Calabi-Yau", arXiv:hep-th/0003263.

[16] M. R. Douglas, B. R. Greene, and D. R. Morrison, "Orbifold resolution by D-branes," Nucl. Phys. B506 (1997) 84, [arXiv:hep-th/9704151].

[17] M. R. Douglas and G. Moore, "D-branes, quivers, and ALE instantons," arXiv:hepth/9603167].

[18] R. Friedman, J. Morgan and E. Witten, "Vector bundles and F theory," Commun. Math. Phys. 187, 679 (1997) [arXiv:hep-th/9701162].

[19] S. Govindarajan, T. Jayaraman, and T. Sarkar, "World sheet approaches to D-branes on supersymmetric cycles", arXiv:hep-th/9907131. 
[20] S. Govindarajan, T. Jayaraman and T. Sarkar, "On D-branes from gauged linear sigma models," Nucl. Phys. B 593, 155 (2001) [arXiv:hep-th/0007075.

[21] S. Govindarajan and T. Jayaraman, "D-branes, exceptional sheaves and quivers on Calabi-Yau manifolds: From Mukai to McKay," Nucl. Phys. B 600, 457 (2001) arXiv:hep-th/0010196.

[22] S. Govindarajan and T. Jayaraman, "Boundary fermions, coherent sheaves and D-branes on Calabi-Yau manifolds," Nucl. Phys. B 618, 50 (2001) arXiv:hepth/0104126.

[23] S. Govindarajan, T. Jayaraman and T. Sarkar, "Disc instantons in linear sigma models," arXiv:hep-th/0108234.

[24] Ph. Griffiths and J. Harris, Principles of algebraic geometry, Wiley \& Sons, 1978.

[25] R. Hartshorne, Algebraic geometry, Springer-Verlag, 1979.

[26] S. Hellerman and J. McGreevy, "Linear sigma model toolshed for D-brane physics," JHEP 0110, 002 (2001) arXiv:hep-th/0104100.

[27] S. Hellerman, S. Kachru, A. E. Lawrence and J. McGreevy, "Linear sigma models for open strings," arXiv:hep-th/0109069.

[28] K. Hori and C. Vafa, "Mirror symmetry," arXiv:hep-th/0002222.

[29] K. Hori, A. Iqbal, and C. Vafa, "D-branes and mirror symmetry", arXiv:hepth/0005247].

[30] V. Kac, "Infinite root systems, representations of graphs and Invariant Theory," Invent. Mathem. 56(1980) 57.

[31] M. Kontsevich and Y. Soibelman, "Homological mirror symmetry and torus fibrations," arXiv:math.sg/0011041.

[32] C. I. Lazaroiu, "String field theory and brane superpotentials," JHEP 0110, 018 (2001) arXiv:hep-th/0107162.

[33] P. Mayr, "Phases of supersymmetric D-branes on Kaehler manifolds and the McKay correspondence," JHEP 0101, 018 (2001) arXiv:hep-th/0010223.

[34] P. Mayr, "N = 1 mirror symmetry and open/closed string duality," arXiv:hepth/0108229]. ;

W. Lerche and P. Mayr, "On N = 1 mirror symmetry for open type II strings," arXiv:hep-th/0111113.

[35] D. R. Morrison and M. Ronen Plesser, "Towards mirror symmetry as duality for two dimensional abelian gauge theories," Nucl. Phys. Proc. Suppl. 46, 177 (1996) arXiv:hep-th/9508107.

[36] J. Mac Cleary, A user's guide to spectral sequences, Cambridge, 2001.

[37] S. Merkulov, "Strong homotopy algebras of a Kähler manifold," arXiv:math.AG/9809172.

[38] B. Mitchell, "Rings with several objects," Adv. Math. 8 (1972) 1-161.

[39] A. Polishchuk, "Homological Mirror Symmetry with Higher Products," arXiv:math.AG/9901025. 
[40] A. Recknagel and V. Schomerus, "D-branes in Gepner models", Nucl. Phys. B531 (1998) 185, arXiv:hep-th/9712186.

[41] M. Reid, "McKay correspondence", arXiv:alg-geom/9702016.

[42] M. Reid, "La correspondance de McKay", Séminaire Bourbaki (novembre 1999), no. 867, arXiv:math.AG/9911165.

[43] P. Seidel, private communication.

[44] P. Seidel and R. Thomas, "Braid group actions on derived categories of sheaves," arXiv:math.AG/0001043.

[45] A. Strominger, S. T. Yau and E. Zaslow, Nucl. Phys. B 479, 243 (1996) arXiv:hepth/9606040].

[46] A. Tomasiello, "A-infinity structure and superpotentials," JHEP 0109, 030 (2001) arXiv:hep-th/0107195.

[47] A. Tomasiello, "D-branes on Calabi-Yau manifolds and helices," JHEP 0102, 008 (2001) arXiv:hep-th/0010217.

[48] E. Witten, "Chern-Simons Gauge Theory as a String Theory," arXiv:hep-th/9207094.

[49] E. Witten, "Phases of $\mathrm{N}=2$ theories in two dimensions," Nucl. Phys. B 403, 159 (1993) arXiv:hep-th/9301042.

[50] E. Witten, "Branes and the Dynamics of QCD", Nucl. Phys. B507, 658 (1997) arXiv:hep-th/9706109. 